\title{
Effective Simple Mathematics Play at Home in Early Childhood: Promoting both Non-cognitive and Cognitive Skills in Early Childhood
}

\author{
Nobuki Watanabe ${ }^{1 *}$ \\ ${ }^{1}$ School of Education, Kwansei Gakuin University, JAPAN \\ *CORRESPONDENCE: $\bigotimes$ nobuki@kwansei.ac.jp
}

\begin{abstract}
In recent years, scholars have increasingly advocated the importance of cultivating non-cognitive skills (social and emotional skills) in early childhood, and play is useful in acquiring the skills. Simultaneously, young children also need to acquire mathematical cognitive skills as a learning foundation. However, many researchers have indicated that simple direct instruction in mathematics is not useful during early education. Therefore, in early childhood, children need forms of play for mathematics at home because play effectively develops non-cognitive skills; moreover, cognitive skills and non-cognitive skills cross-fertilize each other. Moreover, in kindergartens and nursery schools, it is difficult to deal with many "maths" (both temporally and content-wise). Also, parental engagement and attachment have considerable impact on noncognitive skills. In this research, we created a form of simple mathematics quiz game (simple mathematics play) that young children can play at home. The main aspect of this play's content is the application of systematic and extensive mathematics in early childhood, without promoting only cognitive skills as a primary objective. And we tried the play for one child as a case study. This case study suggests that certain improvements in cognitive skills regarding mathematics were recognized because of play, while also enabling acquisition of non-cognitive skills.
\end{abstract}

Keywords: non-cognitive skills, cognitive skills, simple mathematics play at home, early childhood

\section{INTRODUCTION}

Many researchers and practitioners have indicated that, in early childhood, learning is a basic part of character formation (see Ministry of Education, Culture, Sports, Science and Technology, 2017). In recent years, scholars have also discussed the importance of cultivating non-cognitive skills in early childhood learning, and many countries' schools have put the theory into practice (Crehan, 2018; Heckman, 2013; OECD, 2015). It is clear that cognitive and non-cognitive skills cross-fertilize each other, that those with higher levels of non-cognitive skills show higher health returns as cognitive skills increase, and that those with higher levels of non-cognitive skills demonstrate faster development of cognitive skills (OECD, 2015). The learning of noncognitive skills is centered on play instead of unidirectional teaching of knowledge. However, the core of such play depends on the interests of the children, and it is preferable if they become actively immersed in these plays (Hirsh-Pasek \& Golinkoff, 2003).

A definition of non-cognitive skills (social and emotional skills) is "individual capacities that can be (a) manifested in consistent patterns of thoughts, feelings, and behaviors, (b) developed through formal and informal learning experiences, and (c) important drivers of socioeconomic outcomes throughout the

Article History: Received 18 December $2018 \bullet$ Revised 20 February $2019 \bullet$ Accepted 21 February 2019

(C) 2019 by the authors; licensee Modestum Ltd., UK. Open Access terms of the Creative Commons Attribution 4.0 International License (http://creativecommons.org/licenses/by/4.0/) apply. The license permits unrestricted use, distribution, and reproduction in any medium, on the condition that users give exact credit to the original author(s) and the source, provide a link to the Creative Commons license, and indicate if they made any changes. 
individual's life" (OECD, 2015). It is also said that families, schools, and communities matter for the development of non-cognitive skills and that parental engagement and attachment have considerable impact on children's early social and emotional development (OECD, 2015). Simultaneously, the study of mathematics during the early years is foundational for future learning. While some have found that, in early childhood, direct instruction in mathematics education develops cognitive skills, many have also indicated that such education has not been effective in the long run (Crehan, 2018; Heckman, 2013). But, it is also pointed out that mathematics learning has a positive influence later in life (Klibanoff, Levine, Huttenlocher, Vasilyeva, \& Hedges, 2006; Siraj-Blatchford, 2015). In early childhood education in Japan, there is little mathematical content (Ministry of Education, Culture, Sports, Science and Technology, 2017) because there are no "subjects" in kindergartens and nursery school. However, mathematics is often studied in tutoring schools or at home under the initiative of parents or guardians. In such cases, the focus is on entrance examinations in middle school, high school, and university, as well as in a section of elementary schools. Considering this situation, it is thought that nurturing the cognitive skills of mathematics is necessary, but considering the characteristics of the development of children, it is unlikely that focusing solely on developing the cognitive skills of mathematics is not very good. If anything, it can be said that fostering cognitive skills in mathematics is desirable along with non-cognitive skills. That is because the effect of "cross-fertilize" is expected. Therefore, play that can provide an experience of mathematics is important. Further, it goes without saying that it is desirable to experience systematic and extensive mathematics.

So, the question arises: what form would this mathematics learning take? First, children should become immersed in mathematical play. By doing so, their non-cognitive skills will develop, and by experiencing mathematics learning, their cognitive skills will develop as well. In such immersive play, children's active interest is, indeed, necessary. Therefore, how does children's interest in mathematics arise? If one considers children as "blank slates," one would have to consider that their interest depends entirely on the environment created by the children's caregivers. In contrast, if genetic effect were strong, one can assume that a certain proportion of children would like mathematics and would be interested in such play. Today, however, genetics and environment are generally seen as mutually influential on gene-environment interaction (Asbury \& Plomin, 2014; Marcus, 2004; Rutter, 2006; Spector, 2012). If this is the case, it is important to manifest genetic influence through the environment, by providing a reasonable level of stimulus. In either case, creating an appropriate environment is important to help children develop a strong interest in mathematics.

Furthermore, efforts at home are important to extend the non-cognitive skills. That is because the "attachment" effect is expected. Currently, it is suggested that "attachment" causes epigenetics (Spector, 2012). On the other hand, if we carefully practice mathematics play in a wide range of areas, we believe that efforts at home will be more effective than those at school. Therefore, it can be thought that simple mathematics play (including broad and systematic mathematics) that parents could practice at home is very effective in nurturing non-cognitive skills and cognitive skills. However, in order for it to be done at home, it must be simpler mathematics play.

Although there is extant research on play in mathematics education and learning mathematics through play, there are few studies on attempts to nurture both non-cognitive skills and cognitive skills simultaneously at home. There are few studies that are conscious of the systematicity and continuity of mathematics learning contents and of its application in wide-ranging multiple areas at home as well (Anders \& Rossbach, 2015; Carpenter, Franke, Johnson, Turrou, \& Wager, 2016; Clements \& Sarama, 2014; Tucker, 2014; Van Hoorn, Nourot, Scales, \& Alward, 2014; Verdine et al., 2014).

A hypothesis of this research is as follows. We can create a simple mathematics early childhood play. In addition, contents of the play can consist of wide-ranging mathematics. And young children can play at home. The play can promote non-cognitive skills and mathematical cognitive skills, because play promote noncognitive skills and contents of the play included mathematics. We will use a single case study to support this hypothesis. It has already been suggested that a single case study is effective (Barlow, Nock, \& Hersen, 2009). Then, the evaluation of cognitive skills is possible from the mathematical perspective. Conversely, it has already been pointed out that non-cognitive skills can be evaluated (OECD, 2015). However, there are various powers with regard to non-cognitive skills. In this work of research, we assume that it is done at home; hence, we focus on the "ability to achieve goal (perseverance) and the ability to control emotions," with the former being the record of administration, and with the latter pertains to psychology. It can be said that the evaluation is possible using a test (for example, a marshmallow test (Mischel, 2014)). 


\section{MATERIALS AND METHODS}

\section{Overview}

This was a longitudinal study involving a single participant at home. The data collection of this study spanned over three years; it was conducted between September 22, 2014 and March 21, 2018 (3years 0month old -6years 5months old), covering the period from the time the participant was three years of age till the time the participant entered elementary school. First, a simple mathematics play (simple mathematics quiz game) was created for the participant. The quiz game was able to be played easily by young children with their parents. Second, this play was conducted continuously with the participant. However, it should be noted that mathematics was never taught explicitly. For instance, we did not teach the participant the correct answers or correct the participant's wrong answers. In addition, when the child answered questions, the parent praised the participant for the response. Regardless of whether the answer was correct or not, we praised to answer. It was observed that the infant seemed to enjoy mathematics, and so do not require direct instruction. The evaluation method was as follows: (1) the development of mathematical cognitive skills in the participant was assessed from answers to quizzes; (2) the marshmallow test was used to analyze the participant's self-control (non-cognitive skills). Kindergarten attendance was used to analyze the participant's GRIT (non-cognitive skills), and (3) as reference, the entrance examination for elementary school was used to comprehensively assess both non-cognitive and cognitive skills.

\section{Participant}

There was only one participant in this study. As a result, this was a single case study. It is pointed out that single case studies are worthwhile as a method of research and can contribute sufficiently to experimental pursuit. (Barlow, Nock, \& Hersen, 2009). The female was three years old at the beginning of the study and was six years and 5 months old by the end of the study. The participant was selected due to the ease of securing sufficient investigation time, and rapport having already been developed. Therefore, informed consent was obtained from both parents. The investigation was conducted at home, and many of the materials used for the quiz originated in the home and the child was able to play freely. The materials included commercially available stationery supplies (compass, triangular ruler, triangular scale, ruler, etc.), commercially available drills, commercially available puzzles (two-dimensional and three-dimensional), commercially available building blocks (Lego, Pythagoras puzzles, etc.), commercially available measuring instruments (scale, thermometer, body temperature thermometer, weighing scale, watch, water temperature thermometer, air temperature thermometer, etc.), commercially available 100-ball abacus, commercially available multiplication table CDs, commercially available 3-D models, commercially available fraction puzzles, and commercially available tablets (with, for example, programing software installed). All of the aforementioned materials are readily available in stationary stores. In Japan, infants can begin to attend kindergarten when they are three years old. However, kindergartens in Japan do not specifically provide mathematics education. Moreover, there is a high possibility that parents who are not aware of the importance of mathematical play in a child's development have not purchased them. Therefore, it is desirable that these items should be in each household, because all of the aforementioned materials are readily available in stationary stores.

\section{Materials \\ Simple mathematics play (simple mathematics quiz game) and evaluation of cognitive skills}

When developing a form of simple mathematics play, that is, a non-cognitive skills-developing play that aims to cultivate cognitive skills, we searched for existing non-cognitive skills development teaching materials and incorporated mathematics learning into them. The materials were as follows: communication with care providers is important in children's plays, as is care providers' ability to conduct play. Examples include riddles and quizzes. For example, at Japanese kindergartens and nursery schools, word plays are used to aid language development (Narita, 2010; Tagami, \& Takaara, 2016). Thus, we decided to create a simple mathematics play that was based on a quiz game. Therefore, the concrete content of the simple mathematics play used in this study is a simple mathematics quiz (game). Since mathematical contents in the play should be systematic and extensive, we selected the contents of this quiz from the contents that need to be learned in the lower grade of Japanese elementary school. Some of the necessary content includes notions that are not learned directly but are basic to mathematics. The reason for this is the necessity of maintaining mathematical systematicity and breadth. In general, Japanese elementary school textbooks maintain systematicity widely. In addition, because it is conscious of what can be solved by young children thinking it is basic. In addition, we utilize 
Table 1. Detailed classification of each area

\begin{tabular}{|c|c|c|}
\hline \multicolumn{3}{|c|}{ N: Numbers and Quantities } \\
\hline \multirow[t]{13}{*}{$\mathrm{N}-1$} & \multicolumn{2}{|c|}{ Concept of Numbers } \\
\hline & $\mathrm{N}-1-1$ & Digit span [ascending order] (counting using natural numbers) \\
\hline & $\mathrm{N}-1-2$ & Digit span [ascending order (partway through)] (counting from a certain natural number up to 10) \\
\hline & $\mathrm{N}-1-3$ & Digit span [descending order] (count down using natural numbers from a number below 10) \\
\hline & $\mathrm{N}-1-4$ & Reading numbers (reading natural numbers) \\
\hline & $\mathrm{N}-1-5$ & Writing numbers (write down natural numbers after hearing them) \\
\hline & $\mathrm{N}-1-6$ & Reading fractions (1/2-1/10 unit fractions) \\
\hline & $\mathrm{N}-1-7$ & Unit fractions \\
\hline & $\mathrm{N}-1-8$ & Ordinary numbers \\
\hline & $\mathrm{N}-1-9$ & Cardinal number \\
\hline & $\mathrm{N}-1-10$ & Comparing the size of cardinal numbers (same element) \\
\hline & $\mathrm{N}-1-11$ & Comparing the size of cardinal numbers (different element) \\
\hline & $\mathrm{N}-1-12$ & Comparing natural numbers \\
\hline \multirow[t]{11}{*}{$\mathrm{N}-2$} & \multicolumn{2}{|c|}{ Addition and subtraction } \\
\hline & $\mathrm{N}-2-1$ & Meaning of "combine" \\
\hline & $\mathrm{N}-2-2$ & Meaning of "increase" \\
\hline & $\mathrm{N}-2-3$ & "O+O": calculations (easy calculation; 1 digit calculation) \\
\hline & $\mathrm{N}-2-4$ & Commutative law (easy meaning) \\
\hline & $\mathrm{N}-2-5$ & Meaning of "decrease" \\
\hline & $\mathrm{N}-2-6$ & Meaning of "difference" \\
\hline & $\mathrm{N}-2-7$ & “O-O” calculations (easy calculation;1-digit calculation) \\
\hline & $\mathrm{N}-2-8$ & Mixed addition and subtraction \\
\hline & $\mathrm{N}-2-9$ & Addition and subtraction calculations that include “व" \\
\hline & $\mathrm{N}-2-10$ & Numerical decomposition \\
\hline \multirow[t]{2}{*}{$\mathrm{N}-3$} & \multicolumn{2}{|c|}{ Multiplication } \\
\hline & $\mathrm{N}-3-1$ & Multiplication table calculations \\
\hline $\mathrm{N}-4$ & \multicolumn{2}{|c|}{ Quantities } \\
\hline & $\mathrm{N}-4-1$ & $\begin{array}{l}\text { Comparing quantities (long-short, high-low [long-short], deep-shallow, fat-thin, higher-lower } \\
\text { [location], wide-narrow, large-small, heavy-light) }\end{array}$ \\
\hline & $\mathrm{N}-4-2$ & $\begin{array}{l}\text { Measuring quantities [scale in kilograms (digital), clock in hours and minutes (analog, digital), body } \\
\text { temperature thermometer in degrees (digital), scale in grams (analog)] }\end{array}$ \\
\hline & $\mathrm{N}-4-3$ & Conservation (number, length, liquid) \\
\hline \multicolumn{3}{|c|}{ G: Geometry } \\
\hline & & $\begin{array}{l}\text { Names of two-dimensional figures (circle, parallelogram, triangle, square, pentagon, hexagon, oval, } \\
\text { octagon, trapezoid, rhombus, rectangle) }\end{array}$ \\
\hline & & $\begin{array}{l}\text { Names of three-dimensional (3D) shapes (cube, rectangular parallelepiped, triangular prism, } \\
\text { triangular pyramid, cylinder, cone, hexagonal prism, sphere, and circular truncated cone) }\end{array}$ \\
\hline & G-3 & $\begin{array}{l}\text { Similarity between 3D shapes (cube, rectangular parallelepiped, triangular prism, triangular pyramid, } \\
\text { cylinder, cone, and hexagonal prism) }\end{array}$ \\
\hline & G-4 & $\begin{array}{l}\text { 3D objects and these developments (cube, rectangular parallelepiped, triangular prism, triangular } \\
\text { pyramid, cylinder, cone, and hexagonal prism) }\end{array}$ \\
\hline & G-5 & Absolute position and relative position (top, bottom, left, right, front, and back) \\
\hline & G-6 & Line symmetry \\
\hline & G-7 & $\begin{array}{l}\text { Drawing figures (freehand [circle, oval, triangle, rectangle, square, rhombus, trapezoid, and } \\
\text { parallelogram) [drawing implements (compass, triangular ruler, ruler, or triangular scale)] }\end{array}$ \\
\hline & G-8 & Drawing development of $3 \mathrm{D}$ objects \\
\hline \multicolumn{3}{|c|}{ L: Language } \\
\hline & L-1 & Transitive law \\
\hline & L-2 & Logic (AND, OR, NOT) \\
\hline & L-3 & Cause-effect relationships \\
\hline
\end{tabular}

everyday items before learning. The contents were classified into three main areas: numbers and quantities $(\mathrm{N})$, geometry $(\mathrm{G})$, and language (L). Each area was classified as shown in Table 1. Also, as quizzes can easily be taken at home, the questions are easy. Specific examples of the questions in the mathematics quiz are shown in Table 2. In addition, since the contents were representative ones, there were the other contents which were handled on a daily life play in this study.

\section{Evaluation of non-cognitive skills (Marshmallow test and attendance status of kindergarten)}

Measuring non-cognitive skills can be reliably done while being challenging (OECD, 2015). However, there are various powers with regard to non-cognitive skills. In this research work, we assume that it is done at home; hence, we focus on the ability to achieve goal (perseverance) and the ability to control emotions, the 
Table 2. Specific examples of questions in the mathematics quiz

\begin{tabular}{|c|c|c|}
\hline \multicolumn{3}{|c|}{ N: Numbers and Quantities } \\
\hline \multirow[t]{15}{*}{$\mathrm{N}-1$} & \multicolumn{2}{|c|}{ Concept of Numbers } \\
\hline & $\mathrm{N}-1-1$ & Start with the number 1 . Let's count as much as possible. \\
\hline & $\mathrm{N}-1-2$ & Can you count from $3(1,2,4,5,6,7,8,9)$ to $10 ?$ \\
\hline & $\mathrm{N}-1-3$ & Can you count from $6(2,3,4,5,7,8,9)$ down to 1 \\
\hline & $\mathrm{N}-1-4$ & [Show 12.] What number is this? \\
\hline & $\mathrm{N}-1-5$ & Can you write down the number twelve? \\
\hline & $\mathrm{N}-1-6$ & [Show $1 / 2$.$] How do you read this?$ \\
\hline & $\mathrm{N}-1-7$ & If you collect three $1 / 5$ ths, how much do you have? \\
\hline & $\mathrm{N}-1-8$ & [Show a set of things lined up in a row.] What number is this from the right? \\
\hline & $\mathrm{N}-1-9$ & [Show a collection of things.] What is the total number of these? \\
\hline & $\mathrm{N}-1-10$ & [Show two sets comprising a different number of balls.] Which one is more? \\
\hline & $\mathrm{N}-1-11$ & [Show, for example, three oranges and two watermelons.] Which one is more? \\
\hline & $\mathrm{N}-1-12$ & [Show 12 and 21.] Which number is bigger? \\
\hline & $\mathrm{N}-1-13$ & [Show a $1 / 2$ and $1 / 5$ piece.] Which one is bigger? \\
\hline & $\mathrm{N}-1-14$ & Which is bigger, 3 or 7 ? \\
\hline \multirow[t]{11}{*}{$\mathrm{N}-2$} & \multicolumn{2}{|c|}{ Addition and subtraction } \\
\hline & $\mathrm{N}-2-1$ & If you combine two oranges and three oranges, how many oranges are there? \\
\hline & $\mathrm{N}-2-2$ & You had three oranges. Then the oranges increased by two. How many oranges do you have in total? \\
\hline & $\mathrm{N}-2-3$ & Can you do $3+2 ?$ \\
\hline & $\mathrm{N}-2-4$ & $3+2=5$ and $2+3=5$. Why are these the same? \\
\hline & $\mathrm{N}-2-5$ & You had five oranges. Then, the oranges decreased by two. How many oranges do you have left? \\
\hline & N-2-6 & $\begin{array}{l}\text { [Show three red balls and five yellow balls.] What is the difference between the balls? Which type of } \\
\text { ball is greater in number? }\end{array}$ \\
\hline & $\mathrm{N}-2-7$ & Can you do $5-2 ?$ \\
\hline & $\mathrm{N}-2-8$ & Can you do $3+5-2$ ? Can you do $5-2+3$ ? \\
\hline & $\mathrm{N}-2-9$ & [Show $\square+2=3$ ] What goes in the $\square$ ? \\
\hline & $\mathrm{N}-2-10$ & 10 is made of 4 and what other number? \\
\hline \multirow[t]{2}{*}{$\mathrm{N}-3$} & \multicolumn{2}{|c|}{ Multiplication } \\
\hline & $\mathrm{N}-3-1$ & $1 \times 1 ? 1 \times 2 ? \ldots 9 \times 9 ?$ [ask in order] \\
\hline $\mathrm{N}-4$ & \multicolumn{2}{|c|}{ Quantities } \\
\hline & N-4-1 & [Show two things.] Which one is \\
\hline & $\mathrm{N}-4-2$ & [When having measured something] How (What) does it say? \\
\hline & $\mathrm{N}-4-3$ & [Piaget's Task] Which one is ○०? \\
\hline \multicolumn{3}{|c|}{ G: Geometry } \\
\hline & G-1 & [Show a shape.] What is the name of this figure? \\
\hline & G-2 & [Show a shape.] What is the name of this? \\
\hline & G-3 & $\begin{array}{l}\text { [Place two sets of similar three-dimensional (3D) shapes in front of the child.] Which ones are related } \\
\text { to the other? }\end{array}$ \\
\hline & G-4 & [Place these developments and 3D shapes in front of the child.] Which ones are sets? \\
\hline & G-5 & Which one is on the right? \\
\hline & G-6 & What kind of shape was opened up to make this shape? \\
\hline & G-7 & $\begin{array}{l}\text { Try drawing a ○○. } \\
\text { [Place a drawing implement (compass, triangular ruler, ruler, or triangular scale) in front of the } \\
\text { child.] Draw a shape with this. }\end{array}$ \\
\hline & G-8 & [Show a three-dimensional shape.] Try drawing the shape. \\
\hline \multicolumn{3}{|c|}{ L: Language } \\
\hline & $\mathrm{L}-1$ & [Show a scene.] What is the order? \\
\hline & $\mathrm{L}-2$ & [Place things in front of the child.] Please pick up $\bigcirc \bigcirc$ and $\bigcirc \bigcirc$. \\
\hline & $\mathrm{L}-3$ & Count the instances of naturally uttered "Why?" in one day. \\
\hline
\end{tabular}

former is the record of administrative of kindergarten, the latter is psychology test. It can be said that the evaluation is possible using a test (for example, a marshmallow test). The basic marshmallow test procedure is as follows. Child subjects are asked to sit in a chair, which is in a room with only desks and chairs and no distracting elements. There is a plate on the desk with a single marshmallow. The experimenter says, "I have one marshmallow. I will leave the room for 15 minutes from now. While I am away, if you do not eat the marshmallow, I will give another marshmallow. If you eat, there will be no more marshmallows."

\section{Evaluation of general skills (cognitive skills and non-cognitive skills)}

Entrance examinations in public and private elementary school in Japan can be used to measure individuals' general abilities. In such entrance examinations, certain kinds of tests are conducted. Entrance 
examinations comprise paper tests covering language, sociability, numbers/shapes, environment, expression, physical functions, and daily life habits, as well as questions involving manipulating physical objects or providing oral answers. Examples include memory, knowledge, common sense, language, quantities, shapes, reasoning, experiments, instructed behavior, meditation, helping, pictures, physical activity (foot race, balance beam, moving balls, jumping, marching, etc.), group behavior, and interviews, among many others. However, the specific characteristics of entrance examinations depend on the school. Elementary school entrance examinations were conducted in the academic year preceding enrollment from approximately October to February for one to two days (the new academic year starts in April in Japan).

\section{Procedures and Data Analysis}

\section{Simple mathematics play (simple mathematics quiz game) and evaluation of cognitive skills}

The simple mathematics quiz game was done once every month each questions. No time limit was set on when the participant could reply. The participant's answers were recorded as there were. No correct answers were taught. In addition, when the child answered questions, the parent praised her for the response. Regardless of whether the answer was correct or not, I praised to answer. When the participant asked questions, ideas and hints were provided accordingly. In addition, if the participant, midway through the play, stated "I do not want to do it," the play was stopped. If the participant agreed to try again, the quiz was done again. Regarding question items, contents that could be judged as workable were added as required. Answers to the quiz questions (N-1-1 L-3) were given in the month the child was A years and B months old. The answers are referred to as "A years, B months stage answers."

In the result table, " $O$ " is correct answers, " $X$ " is incorrect answers, and " $\triangle$ " is partial correct answers, etc. In the case where it is impossible to be able to understand the question or to ask the question (we had no time), it is indicated as "an oblique line." In addition, the participant's cognition was highlighted whenever it improved.

\section{Evaluation of non-cognitive skills (Marshmallow test and attendance status of kindergarten)}

The marshmallow test is 15 minutes long. If the subject does not eat the marshmallow, they clear the test. Attendance status of kindergarten is a record of whether or not a child attended kindergarten.

\section{Evaluation of general skills (Cognitive Skills and non-cognitive skills)}

Many elementary schools' exams are conducted over a period of two days. It takes about 15 minutes by interview. Writing and behavior test took about 4-5 hours. Passing the test was regarded as clear.

\section{RESULTS}

\section{Simple Mathematics Play (Simple Mathematics Quiz Game) and Evaluation of Cognitive Skills}

The results are shown in Table 3. 
Table 3. Results of the mathematics quiz

\begin{tabular}{|c|c|c|c|c|c|c|c|c|c|c|c|c|}
\hline & $\mathrm{N}-1-1$ & $\mathrm{~N}-1-2$ & $\mathrm{~N}-1-3$ & $\mathrm{~N}-1-4$ & $\mathrm{~N}-1-5$ & $\mathrm{~N}-1-6$ & $\mathrm{~N}-1-7$ & $\mathrm{~N}-1-8$ & $\mathrm{~N}-1-9$ & $\mathrm{~N}-1-10$ & $\mathrm{~N}-1-11$ & N-1-12 \\
\hline 3years 0month & 15 & 7 & 7 & $0 \sim 9$ & 1 & 1 & & 2 & (until) 9 & 0 & $x$ & 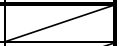 \\
\hline 3years 1month & 20 & 7 & 7 & $0 \sim 13$ & $>$ & 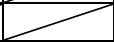 & & $x$ & 10 & $\uparrow$ & $\uparrow$ & 7 \\
\hline 3years 2months & 19 & 7 & 7 & $\uparrow$ & 1 & 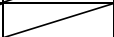 & & $x$ & 20 & $\uparrow$ & 7 & 7 \\
\hline 3years 3months & 39 & 1 & 1 & $\uparrow$ & $1 \sim 10$ & $1 / 2-1 / 10$ & & 0 & 29 & $\uparrow$ & $x$ & $x$ \\
\hline 3years 4months & 69 & $\triangle$ & $10 \rightarrow 0$ & $0 \sim 20$ & $\uparrow$ & $\uparrow$ & & $\uparrow$ & 69 & $\uparrow$ & $\uparrow$ & $\uparrow$ \\
\hline 3years 5months & $\uparrow$ & $\uparrow$ & $\uparrow$ & $\uparrow$ & $\uparrow$ & $\uparrow$ & & $\uparrow$ & $\uparrow$ & $\uparrow$ & 0 & $\uparrow$ \\
\hline 3years 6months & $\uparrow$ & $\uparrow$ & $\uparrow$ & $\uparrow$ & $\uparrow$ & $\uparrow$ & & $\uparrow$ & $\uparrow$ & $\uparrow$ & $\uparrow$ & $\uparrow$ \\
\hline 3years 7months & $\uparrow$ & $\uparrow$ & $\uparrow$ & $\uparrow$ & $\uparrow$ & $\uparrow$ & & $\uparrow$ & $\uparrow$ & $\uparrow$ & $\uparrow$ & $\triangle$ \\
\hline 3years 8months & $\uparrow$ & $\uparrow$ & $\uparrow$ & $\uparrow$ & $\uparrow$ & $\uparrow$ & & $\uparrow$ & $\uparrow$ & $\uparrow$ & $\uparrow$ & $\uparrow$ \\
\hline 3years 9months & 59 & $\uparrow$ & $\uparrow$ & $\uparrow$ & $\uparrow$ & $\uparrow$ & & $\uparrow$ & 59 & $\uparrow$ & $\uparrow$ & $1 \sim 10$ \\
\hline 3years 10months & 60 & $\uparrow$ & $\uparrow$ & $0 \sim 15$ & $\uparrow$ & $\uparrow$ & & $\uparrow$ & 60 & $\uparrow$ & $\uparrow$ & $\uparrow$ \\
\hline 3years 11months & $\uparrow$ & $\uparrow$ & $\uparrow$ & $0 \sim 20$ & $\uparrow$ & $\uparrow$ & & $\uparrow$ & $\uparrow$ & $\uparrow$ & $\uparrow$ & $\uparrow$ \\
\hline 4years 0month & $\uparrow$ & $\uparrow$ & $\uparrow$ & $\uparrow$ & $\uparrow$ & $\uparrow$ & & $\uparrow$ & $\uparrow$ & \begin{tabular}{|c} 
Range \\
of digit \\
span \\
0 \\
\end{tabular} & $\uparrow$ & $\uparrow$ \\
\hline 4years 1month & 100 & $\uparrow$ & $\uparrow$ & $\uparrow$ & $1 \sim 15$ & $\uparrow$ & & $\uparrow$ & $\begin{array}{c}\text { Range } \\
\text { of digit } \\
\text { span } \\
0\end{array}$ & $\uparrow$ & $\uparrow$ & $\uparrow$ \\
\hline 4years 2months & 111 & $\uparrow$ & $\uparrow$ & $\uparrow$ & $1 \sim 10$ & $\uparrow$ & & $\uparrow$ & $\uparrow$ & $\uparrow$ & $\uparrow$ & $\uparrow$ \\
\hline 4years 3months & 100 & $\uparrow$ & $\uparrow$ & $\uparrow$ & $\uparrow$ & $\uparrow$ & & $\uparrow$ & $\uparrow$ & $\uparrow$ & $\uparrow$ & $\uparrow$ \\
\hline 4years 4months & $\uparrow$ & $\uparrow$ & $\uparrow$ & $\uparrow$ & $\uparrow$ & $\uparrow$ & & $\uparrow$ & $\uparrow$ & $\uparrow$ & $\uparrow$ & $\uparrow$ \\
\hline 4years 5months & 95 & $\uparrow$ & $\begin{array}{c}(1 \sim 10) \\
\rightarrow 0 \\
\triangle\end{array}$ & $\uparrow$ & $1 \sim 19$ & $\uparrow$ & & $\uparrow$ & $\uparrow$ & $\uparrow$ & $\uparrow$ & $\uparrow$ \\
\hline 4years 6months & 100 & 0 & $\uparrow$ & $0 \sim 99$ & $\uparrow$ & $\uparrow$ & & $\uparrow$ & $\uparrow$ & $\uparrow$ & $\uparrow$ & $\uparrow$ \\
\hline 4years 7months & $\uparrow$ & $\triangle$ & $\uparrow$ & $\uparrow$ & $\uparrow$ & $\uparrow$ & & $\uparrow$ & $\uparrow$ & $\uparrow$ & $\uparrow$ & $\uparrow$ \\
\hline 4years 8months & $\uparrow$ & $\uparrow$ & 0 & $0 \sim 100$ & $\uparrow$ & $\uparrow$ & 4 & $\uparrow$ & $\uparrow$ & $\uparrow$ & $\uparrow$ & $1 \sim 20$ \\
\hline 4years 9months & $\uparrow$ & O & $\uparrow$ & $\uparrow$ & $\uparrow$ & $\uparrow$ & 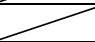 & $\uparrow$ & $\uparrow$ & $\uparrow$ & $\uparrow$ & $\uparrow$ \\
\hline 4years 10months & $\uparrow$ & $\uparrow$ & $\uparrow$ & $\uparrow$ & $1 \sim 50$ & $\uparrow$ & & $\uparrow$ & $\uparrow$ & $\uparrow$ & $\uparrow$ & $\uparrow$ \\
\hline 4years 11months & $\uparrow$ & $\uparrow$ & $\uparrow$ & $\uparrow$ & $1 \sim 30$ & $\uparrow$ & 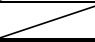 & $\uparrow$ & $\uparrow$ & $\uparrow$ & $\uparrow$ & $1 \sim 30$ \\
\hline 5years 0month & 139 & $\uparrow$ & $\uparrow$ & $\uparrow$ & $1 \sim 39$ & $\uparrow$ & $\mathrm{O}$ & $\uparrow$ & $\uparrow$ & $\uparrow$ & $\uparrow$ & $1 \sim 20$ \\
\hline 5years 1month & 100 & $\uparrow$ & $\uparrow$ & $\uparrow$ & $\uparrow$ & $\uparrow$ & $\uparrow$ & $\uparrow$ & $\uparrow$ & $\uparrow$ & $\uparrow$ & $1 \sim 100$ \\
\hline 5years 2 months & $\uparrow$ & $\uparrow$ & $\uparrow$ & $\uparrow$ & $1 \sim 50$ & $\uparrow$ & $\uparrow$ & $\uparrow$ & $\uparrow$ & $\uparrow$ & $\uparrow$ & $1 \sim 30$ \\
\hline 5years 3months & $\uparrow$ & $\uparrow$ & $\uparrow$ & $1 \sim 199$ & $1 \sim 30$ & $\uparrow$ & $\uparrow$ & $\uparrow$ & $\uparrow$ & $\uparrow$ & $\uparrow$ & $1 \sim 100$ \\
\hline 5years 4 months & $\uparrow$ & $\uparrow$ & $\uparrow$ & $1 \sim 999$ & $1 \sim 70$ & $\uparrow$ & $\uparrow$ & $\uparrow$ & $\uparrow$ & $\uparrow$ & $\uparrow$ & $1 \sim 999$ \\
\hline 5years 5months & $\uparrow$ & $\uparrow$ & $\uparrow$ & $\uparrow$ & $\uparrow$ & $\uparrow$ & $\uparrow$ & $\uparrow$ & $\uparrow$ & $\uparrow$ & $\uparrow$ & $\uparrow$ \\
\hline 5 years 6months & 120 & $\uparrow$ & $\uparrow$ & $\uparrow$ & $1 \sim 43$ & $\uparrow$ & $\uparrow$ & $\uparrow$ & $\uparrow$ & $\uparrow$ & $\uparrow$ & $\uparrow$ \\
\hline 5years 7months & 100 & $\uparrow$ & $\uparrow$ & $1 \sim 199$ & $1 \sim 80$ & $\uparrow$ & $\uparrow$ & $\uparrow$ & $\uparrow$ & $\uparrow$ & $\uparrow$ & $1 \sim 200$ \\
\hline 5years 8months & 102 & $\uparrow$ & $\uparrow$ & $\uparrow$ & $1 \sim 99$ & $\uparrow$ & $\uparrow$ & $\uparrow$ & $\uparrow$ & $\uparrow$ & $\uparrow$ & $\uparrow$ \\
\hline 5years 9months & 110 & $\uparrow$ & $\uparrow$ & $1 \sim 999$ & $1 \sim 100$ & $\uparrow$ & $\uparrow$ & $\uparrow$ & $\uparrow$ & $\uparrow$ & $\uparrow$ & $1 \sim 900$ \\
\hline 5years 10 months & 119 & $\uparrow$ & $\uparrow$ & $\uparrow$ & $\uparrow$ & $\uparrow$ & $\uparrow$ & $\uparrow$ & $\uparrow$ & $\uparrow$ & $\uparrow$ & $\uparrow$ \\
\hline 5 years 11 months & $\uparrow$ & $\uparrow$ & $\uparrow$ & $\uparrow$ & $1 \sim 110$ & $\uparrow$ & $\uparrow$ & $\uparrow$ & $\uparrow$ & $\uparrow$ & $\uparrow$ & $\uparrow$ \\
\hline 6years 0month & 129 & $\uparrow$ & $\uparrow$ & $1 \sim 1000$ & $1 \sim 100$ & $\uparrow$ & $\uparrow$ & $\uparrow$ & $\uparrow$ & $\uparrow$ & $\uparrow$ & $\uparrow$ \\
\hline 6years 1month & 199 & $\uparrow$ & $\uparrow$ & $1 \sim 2000$ & $1 \sim 109$ & $\uparrow$ & $\uparrow$ & $\uparrow$ & $\uparrow$ & $\uparrow$ & $\uparrow$ & $1 \sim 1100$ \\
\hline 6 years 2 months & $\uparrow$ & $\uparrow$ & $\uparrow$ & $\uparrow$ & $1 \sim 119$ & $\uparrow$ & $\uparrow$ & $\uparrow$ & $\uparrow$ & $\uparrow$ & $\uparrow$ & $\uparrow$ \\
\hline 6years 3months & $\uparrow$ & $\uparrow$ & $\uparrow$ & $\uparrow$ & $\uparrow$ & $\uparrow$ & $\uparrow$ & $\uparrow$ & $\uparrow$ & $\uparrow$ & $\uparrow$ & $\uparrow$ \\
\hline 6years 4months & 139 & $\uparrow$ & $\uparrow$ & $\uparrow$ & $1 \sim 135$ & $\uparrow$ & $\uparrow$ & $\uparrow$ & $\uparrow$ & $\uparrow$ & $\uparrow$ & $\uparrow$ \\
\hline 6years 5months & 119 & $\uparrow$ & $\uparrow$ & $\uparrow$ & $1 \sim 199$ & $\uparrow$ & $\uparrow$ & $\uparrow$ & $\uparrow$ & $\uparrow$ & $\uparrow$ & $\uparrow$ \\
\hline
\end{tabular}




\section{Watanabe}

Table 3 (continued). Results of the mathematics quiz

\begin{tabular}{|c|c|c|c|c|c|c|c|c|c|c|}
\hline & $\mathrm{N}-2-1$ & $\mathrm{~N}-2-2$ & $\mathrm{~N}-2-3$ & $\mathrm{~N}-2-4$ & $\mathrm{~N}-2-5$ & $\mathrm{~N}-2-6$ & $\mathrm{~N}-2-7$ & $\mathrm{~N}-2-8$ & $\mathrm{~N}-2-9$ & $\mathrm{~N}-2-10$ \\
\hline 3years 0month & W & W & $\infty$ & & W & 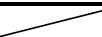 & & & & \\
\hline 3years 1month & 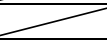 & 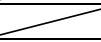 &  & &  & $\infty$ & & & & \\
\hline 3years 2months & 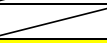 & 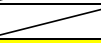 & $\infty$ & & $\infty$ & 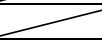 & 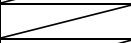 & & & \\
\hline 3years 3months & $\mathrm{O}$ & $\mathrm{O}$ & 1 & & $\infty$ & 1 & 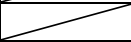 & & & \\
\hline 3years 4months & $\uparrow$ & $\uparrow$ & 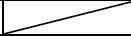 & & 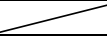 & 1 & $\longrightarrow$ & & & \\
\hline 3years 5months & $\uparrow$ & $\uparrow$ & 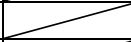 & & $\mathrm{O}$ & $\triangle$ & 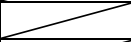 & & & \\
\hline 3years 6months & $\uparrow$ & $\uparrow$ & $\infty$ & 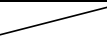 & $\uparrow$ & $\uparrow$ & 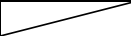 & & & \\
\hline 3years 7months & $\uparrow$ & $\uparrow$ & 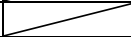 & & $\uparrow$ & $\uparrow$ & $\infty$ & & & \\
\hline 3years 8months & $\uparrow$ & $\uparrow$ & $\begin{array}{l}\mathrm{O} 1+1,2+2, \\
\text { etc. } \\
\text { (Easy } \\
\text { calculation) }\end{array}$ & & $\uparrow$ & $\uparrow$ & & & & \\
\hline 3years 9months & $\uparrow$ & $\uparrow$ & $\uparrow$ & & $\uparrow$ & $\uparrow$ & 7 & & & $1 \sim 3$ \\
\hline 3years 10months & $\uparrow$ & $\uparrow$ & $\uparrow$ & & $\uparrow$ & $\uparrow$ & $\infty$ & & & $1 \sim 4$ \\
\hline 3years 11months & $\uparrow$ & $\uparrow$ & $\uparrow$ & & $\uparrow$ & $\uparrow$ & $\infty$ & & & $\uparrow$ \\
\hline 4years 0month & $\uparrow$ & $\uparrow$ & $\uparrow$ & & $\uparrow$ & 0 & $\infty$ & & & $\uparrow$ \\
\hline 4years 1month & $\uparrow$ & $\uparrow$ & $\uparrow$ & & $\uparrow$ & $\uparrow$ & 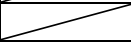 & & & $1 \sim 5$ \\
\hline 4years 2months & $\uparrow$ & $\uparrow$ & $\uparrow$ & & $\uparrow$ & $\uparrow$ & $\infty$ & & & $\uparrow$ \\
\hline 4years 3months & $\uparrow$ & $\uparrow$ & $\uparrow$ & & $\uparrow$ & $\uparrow$ & 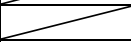 & & & $\uparrow$ \\
\hline 4years 4months & $\uparrow$ & $\uparrow$ & $\begin{array}{c}\mathrm{O} 1+1 \\
4+4+4 \text { etc. } \\
\text { (Easy } \\
\text { calculation) }\end{array}$ & & $\uparrow$ & $\uparrow$ & $x$ & & & $\uparrow$ \\
\hline 4years 5months & $\uparrow$ & $\uparrow$ & $\uparrow$ & & $\uparrow$ & $\uparrow$ & $\infty$ & & 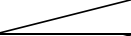 & $\uparrow$ \\
\hline 4years 6months & $\uparrow$ & $\uparrow$ & $\uparrow$ & $\sim$ & $\uparrow$ & $\uparrow$ & $x$ & $\infty$ & $\infty$ & $\uparrow$ \\
\hline 4years 7months & $\uparrow$ & $\uparrow$ & $\uparrow$ & $\sim$ & $\uparrow$ & $\uparrow$ & $\infty$ & $\infty$ & $\infty$ & $\uparrow$ \\
\hline 4years 8months & $\uparrow$ & $\uparrow$ & $\uparrow$ & 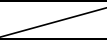 & $\uparrow$ & $\uparrow$ & O3-1etc & 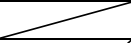 & 4 & $\uparrow$ \\
\hline 4years 9months & $\uparrow$ & $\uparrow$ & $\uparrow$ & & $\uparrow$ & $\uparrow$ & $\begin{array}{c}\text { O3-1,5-2 } \\
\text { etc. } \\
\text { (Easy } \\
\text { calculation) }\end{array}$ & & & $\uparrow$ \\
\hline 4years 10months & $\uparrow$ & $\uparrow$ & $\uparrow$ & $\infty$ & $\uparrow$ & $\uparrow$ & $\uparrow$ & $\infty$ &  & $\uparrow$ \\
\hline 4years 11months & $\uparrow$ & $\uparrow$ & $\uparrow$ & $>$ & $\uparrow$ & $\uparrow$ & $\uparrow$ &  & 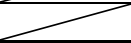 & $1 \sim 5,10$ \\
\hline 5years 0month & $\uparrow$ & $\uparrow$ & $\uparrow$ & 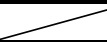 & $\uparrow$ & $\uparrow$ & $\uparrow$ & 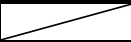 & $\Gamma$ & $1 \sim 5$ \\
\hline 5years 1month & $\uparrow$ & $\uparrow$ & $\uparrow$ & 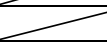 & $\uparrow$ & $\uparrow$ & $\uparrow$ & 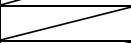 & 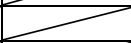 & $\uparrow$ \\
\hline 5years 2months & $\uparrow$ & $\uparrow$ & $\uparrow$ & 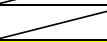 & $\uparrow$ & $\uparrow$ & $\uparrow$ & $\infty$ & $\infty$ & $\uparrow$ \\
\hline 5years 3months & $\uparrow$ & $\uparrow$ & $\uparrow$ & 0 & $\uparrow$ & $\uparrow$ & $\uparrow$ & $\infty$ & 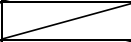 & $\uparrow$ \\
\hline 5years 4months & $\uparrow$ & $\uparrow$ & $\uparrow$ & $\uparrow$ & $\uparrow$ & $\uparrow$ & $\uparrow$ & $\infty$ & $\infty$ & $\uparrow$ \\
\hline 5years 5months & $\uparrow$ & $\uparrow$ & $\uparrow$ & $\uparrow$ & $\uparrow$ & $\uparrow$ & $\uparrow$ & $\infty$ & $\infty$ & $\uparrow$ \\
\hline 5years 6months & $\uparrow$ & $\uparrow$ & $\uparrow$ & $\infty$ & $\uparrow$ & $\uparrow$ & $\uparrow$ & $\infty$ & $\infty$ & $\uparrow$ \\
\hline 5years 7months & $\uparrow$ & $\uparrow$ & $\uparrow$ & 0 & $\uparrow$ & $\uparrow$ & $\uparrow$ & 1 & 1 & $\uparrow$ \\
\hline 5years 8months & $\uparrow$ & $\uparrow$ & $\uparrow$ & $\uparrow$ & $\uparrow$ & $\uparrow$ & $\uparrow$ & $\infty$ & $\infty$ & $\uparrow$ \\
\hline 5years 9months & $\uparrow$ & $\uparrow$ & $\uparrow$ & $\uparrow$ & $\uparrow$ & $\uparrow$ & $\uparrow$ & $\triangle$ & $\infty$ & $\uparrow$ \\
\hline 5years 10months & $\uparrow$ & $\uparrow$ & $\uparrow$ & $\uparrow$ & $\uparrow$ & $\uparrow$ & $\uparrow$ & $\uparrow$ & $\infty$ & $1 \sim 5,10$ \\
\hline 5years 11 months & $\uparrow$ & $\uparrow$ & $\uparrow$ & $\uparrow$ & $\uparrow$ & $\uparrow$ & $\uparrow$ & $\uparrow$ & $\begin{array}{c}\bigcirc \\
\square+3=4 \\
5-\square=2 \\
\text { etc. }\end{array}$ & $1 \sim 5$ \\
\hline 6years 0month & $\uparrow$ & $\uparrow$ & $\uparrow$ & $\uparrow$ & $\uparrow$ & $\uparrow$ & $\uparrow$ & $\begin{array}{c}0 \\
\text { (Easy } \\
\text { calculation) }\end{array}$ & $\uparrow$ & $1 \sim 5,10$ \\
\hline 6years 1month & $\uparrow$ & $\uparrow$ & $\uparrow$ & $\uparrow$ & $\uparrow$ & $\uparrow$ & $\uparrow$ & $\uparrow$ & $\uparrow$ & $\uparrow$ \\
\hline 6years 2months & $\uparrow$ & $\uparrow$ & $\uparrow$ & $\uparrow$ & $\uparrow$ & $\uparrow$ & $\uparrow$ & $\uparrow$ & $\uparrow$ & $\uparrow$ \\
\hline 6years 3months & $\uparrow$ & $\uparrow$ & $\uparrow$ & $\uparrow$ & $\uparrow$ & $\uparrow$ & $\uparrow$ & $\uparrow$ & $\begin{array}{l}\square+3=5 \\
4+\square=6 \\
\square-2=3 \\
3-\square=2\end{array}$ & $\uparrow$ \\
\hline 6years 4months & $\uparrow$ & $\uparrow$ & $\uparrow$ & $\infty$ & $\uparrow$ & $\uparrow$ & $\uparrow$ & $\uparrow$ & $\uparrow$ & $\uparrow$ \\
\hline 6years 5months & $\uparrow$ & $\uparrow$ & $\uparrow$ & $\mathrm{O}$ & $\uparrow$ & $\uparrow$ & $\uparrow$ & $\uparrow$ & $\uparrow$ & $\uparrow$ \\
\hline
\end{tabular}


Table 3 (continued). Results of the mathematics quiz

\begin{tabular}{|c|c|c|c|c|c|c|c|c|c|c|c|c|c|c|c|c|c|}
\hline & \multirow[t]{2}{*}{$\mathrm{N}-3$} & \multicolumn{8}{|c|}{$\mathrm{N}-4-1$} & \multicolumn{5}{|c|}{$\mathrm{N}-4-2$} & \multicolumn{3}{|c|}{$\mathrm{N}-4-3$} \\
\hline & & 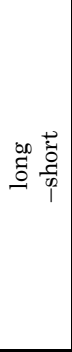 & 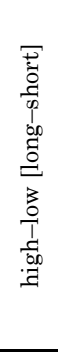 & 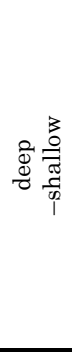 & 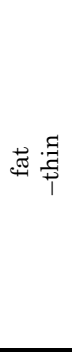 & 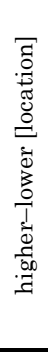 & 总 & 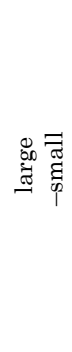 & 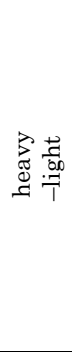 & 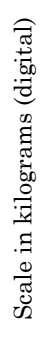 & 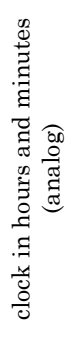 &  & 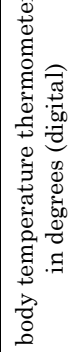 & 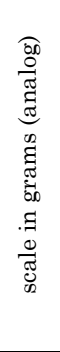 & 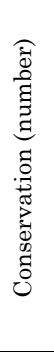 & 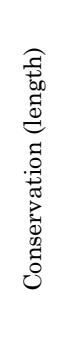 & 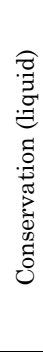 \\
\hline 3years 0month & & 0 & & 7 & $\mathrm{O}$ & 7 & 0 & 0 & 0 & $x$ & $x$ & $x$ & 7 & & & & \\
\hline 3years 1month & & $\uparrow$ & & 7 & $x$ & 0 & $\uparrow$ & $\uparrow$ & $\uparrow$ & $\uparrow$ & $\uparrow$ & $\uparrow$ & & & & 4 & \\
\hline 3 years 2 months & & $\uparrow$ & & $\mathrm{O}$ & O & $\uparrow$ & $\uparrow$ & $\uparrow$ & $\uparrow$ & $\uparrow$ & $\uparrow$ & $\uparrow$ & 4 & 4 & $x$ & $x$ & $x$ \\
\hline 3years 3months & & $\uparrow$ & & $\uparrow$ & $\uparrow$ & $\uparrow$ & $\uparrow$ & $\uparrow$ & $\uparrow$ & $\uparrow$ & $\uparrow$ & $\uparrow$ & $x$ & 1 & $\uparrow$ & $\uparrow$ & $\uparrow$ \\
\hline 3years 4months & & $\uparrow$ & & $\uparrow$ & $\uparrow$ & $\uparrow$ & $\uparrow$ & $\uparrow$ & $\uparrow$ & $\uparrow$ & $\uparrow$ & $\uparrow$ & $\uparrow$ & $\times$ & $\uparrow$ & $\uparrow$ & $\uparrow$ \\
\hline 3years 5months & & $\uparrow$ & & $\uparrow$ & $\uparrow$ & $\uparrow$ & $\uparrow$ & $\uparrow$ & $\uparrow$ & $\uparrow$ & $\uparrow$ & $\uparrow$ & $\uparrow$ & $\uparrow$ & $\uparrow$ & $\uparrow$ & $\uparrow$ \\
\hline 3years 6months & & $\uparrow$ & & $\uparrow$ & $\uparrow$ & $\uparrow$ & $\uparrow$ & $\uparrow$ & $\uparrow$ & $\uparrow$ & $\uparrow$ & $\uparrow$ & $\uparrow$ & $\uparrow$ & $\uparrow$ & 0 & $\uparrow$ \\
\hline 3years 7months & 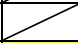 & $\uparrow$ & & $\uparrow$ & $\uparrow$ & $\uparrow$ & $\uparrow$ & $\uparrow$ & $\uparrow$ & $\uparrow$ & $\uparrow$ & $\uparrow$ & $\uparrow$ & $\uparrow$ & 0 & $\uparrow$ & $\mathrm{O}$ \\
\hline 3years 8months & 1 & $\uparrow$ & & $\uparrow$ & $\uparrow$ & $\uparrow$ & $\uparrow$ & $\uparrow$ & $\uparrow$ & $\uparrow$ & $\uparrow$ & $\uparrow$ & $\uparrow$ & $\uparrow$ & $\uparrow$ & $\uparrow$ & $\uparrow$ \\
\hline 3years 9months & $\uparrow$ & $\uparrow$ & & $\uparrow$ & $\uparrow$ & $\uparrow$ & $\uparrow$ & $\uparrow$ & $\uparrow$ & $\uparrow$ & $\uparrow$ & $\uparrow$ & $\uparrow$ & $\uparrow$ & $\uparrow$ & $\uparrow$ & $\uparrow$ \\
\hline 3years 10months & 1,5 & $\uparrow$ & & $\uparrow$ & $\uparrow$ & $\uparrow$ & $\uparrow$ & $\uparrow$ & $\uparrow$ & $\uparrow$ & $\uparrow$ & $\uparrow$ & $\uparrow$ & $\uparrow$ & $\uparrow$ & $\uparrow$ & $\uparrow$ \\
\hline 3years 11months & 1 & $\uparrow$ & & $\uparrow$ & $\uparrow$ & $\uparrow$ & $\uparrow$ & $\uparrow$ & $\uparrow$ & $\uparrow$ & $\uparrow$ & 0 & $\uparrow$ & $\uparrow$ & $\uparrow$ & $\uparrow$ & $\uparrow$ \\
\hline 4years 0month & $1,3,5$ & $\uparrow$ & & $\uparrow$ & $\uparrow$ & $\uparrow$ & $\uparrow$ & $\uparrow$ & $\uparrow$ & $\uparrow$ & $\uparrow$ & $\uparrow$ & $\uparrow$ & $\uparrow$ & $\uparrow$ & $\uparrow$ & $\uparrow$ \\
\hline 4years 1month & 1,5 & $\uparrow$ & & $\uparrow$ & $\uparrow$ & $\uparrow$ & $\uparrow$ & $\uparrow$ & $\uparrow$ & $\uparrow$ & $\uparrow$ & $\uparrow$ & $\uparrow$ & $\uparrow$ & $\uparrow$ & $\uparrow$ & $\uparrow$ \\
\hline 4years 2months & $\uparrow$ & $\uparrow$ & & $\uparrow$ & $\uparrow$ & $\uparrow$ & $\uparrow$ & $\uparrow$ & $\uparrow$ & $\uparrow$ & $\uparrow$ & $\uparrow$ & $\uparrow$ & 0 & $\uparrow$ & $\uparrow$ & $\uparrow$ \\
\hline 4years 3months & $\uparrow$ & $\uparrow$ & & $\uparrow$ & $\uparrow$ & $\uparrow \uparrow$ & $\uparrow$ & $\uparrow$ & $\uparrow$ & $\uparrow \uparrow$ & $\uparrow$ & $\uparrow$ & $\uparrow$ & $\uparrow \uparrow$ & $\uparrow \uparrow$ & $\uparrow$ & $\uparrow \uparrow$ \\
\hline 4years 4months & $\uparrow$ & $\uparrow$ & & $\uparrow$ & $\uparrow$ & $\uparrow$ & $\uparrow$ & $\uparrow$ & $\uparrow$ & $\uparrow$ & $\uparrow$ & $\uparrow$ & $\uparrow$ & $x$ & $\uparrow$ & $\uparrow$ & $\uparrow$ \\
\hline 4years 5months & $\uparrow$ & $\uparrow$ & 4 & $\uparrow$ & $\uparrow$ & $\uparrow$ & $\uparrow$ & $\uparrow$ & $\uparrow$ & $\uparrow$ & $\uparrow$ & $\uparrow$ & $\uparrow$ & $\mathrm{O}$ & $\uparrow$ & $\uparrow$ & $\uparrow$ \\
\hline 4years 6months & $\uparrow$ & $\uparrow$ & 0 & $\uparrow$ & $\uparrow$ & $\uparrow$ & $\uparrow$ & $\uparrow$ & $\uparrow$ & $\uparrow$ & $\uparrow$ & $\uparrow$ & $\mathrm{O}$ & $x$ & $\uparrow$ & $\uparrow$ & $\uparrow$ \\
\hline 4years 7 months & 1 & $\uparrow$ & $\uparrow$ & $\uparrow$ & $\uparrow$ & $\uparrow$ & $\uparrow$ & $\uparrow$ & $\uparrow$ & $\uparrow$ & $\uparrow$ & $\uparrow$ & $\uparrow$ & $\mathrm{O}$ & $\uparrow$ & $\uparrow$ & $\uparrow$ \\
\hline 4years 8months & 1,5 & $\uparrow$ & $\uparrow$ & $\uparrow$ & $\uparrow$ & $\uparrow$ & $\uparrow$ & $\uparrow$ & $\uparrow$ & $\uparrow$ & 0 & $\uparrow$ & $\uparrow$ & $\uparrow$ & $\uparrow$ & $\uparrow$ & $\uparrow$ \\
\hline 4years 9months & 1 & $\uparrow$ & $\uparrow$ & $\uparrow$ & $\uparrow$ & $\uparrow$ & $\uparrow$ & $\uparrow$ & $\uparrow$ & $\uparrow$ & $\uparrow$ & $\uparrow$ & $\uparrow$ & $\uparrow$ & $\uparrow$ & $\uparrow$ & $\uparrow$ \\
\hline 4years 10months & $\uparrow$ & $\uparrow$ & $\uparrow$ & $\uparrow$ & $\uparrow$ & $\uparrow$ & $\uparrow$ & $\uparrow$ & $\uparrow$ & $\uparrow$ & $\uparrow$ & $\uparrow$ & $\uparrow$ & $x$ & $\uparrow$ & $\uparrow$ & $\uparrow$ \\
\hline 4years 11months & $\uparrow$ & $\uparrow$ & $\uparrow$ & $\uparrow$ & $\uparrow$ & $\uparrow$ & $\uparrow$ & $\uparrow$ & $\uparrow$ & $\uparrow$ & $\uparrow$ & $\uparrow$ & $\uparrow$ & $\mathrm{O}$ & $\uparrow$ & $\uparrow$ & $\uparrow$ \\
\hline 5years 0month & $\uparrow$ & $\uparrow$ & $\uparrow$ & $\uparrow$ & $\uparrow$ & $\uparrow$ & $\uparrow$ & $\uparrow$ & $\uparrow$ & $\uparrow$ & $\uparrow$ & $\uparrow$ & $\uparrow$ & $\uparrow$ & $\uparrow$ & $\uparrow$ & $\uparrow$ \\
\hline 5years 1month & $\uparrow$ & $\uparrow$ & $\uparrow$ & $\uparrow$ & $\uparrow$ & $\uparrow$ & $\uparrow$ & $\uparrow$ & $\uparrow$ & $\uparrow$ & $\uparrow$ & $\uparrow$ & $\uparrow$ & $\uparrow$ & $\uparrow$ & $\uparrow$ & $\uparrow$ \\
\hline 5years 2months & $\uparrow$ & $\uparrow$ & $\uparrow$ & $\uparrow$ & $\uparrow$ & $\uparrow$ & $\uparrow$ & $\uparrow$ & $\uparrow$ & $\uparrow$ & $\uparrow$ & $\uparrow$ & $\uparrow$ & $\uparrow$ & $\uparrow$ & $\uparrow$ & $\uparrow$ \\
\hline 5years 3months & $\uparrow$ & $\uparrow$ & $\uparrow$ & $\uparrow$ & $\uparrow$ & $\uparrow$ & $\uparrow$ & $\uparrow$ & $\uparrow$ & $\uparrow$ & $\uparrow$ & $\uparrow$ & $\uparrow$ & $x$ & $\uparrow$ & $\uparrow$ & $\uparrow$ \\
\hline 5years 4months & $\uparrow$ & $\uparrow$ & $\uparrow$ & $\uparrow$ & $\uparrow$ & $\uparrow$ & $\uparrow$ & $\uparrow$ & $\uparrow$ & $\uparrow$ & $\uparrow$ & $\uparrow$ & $\uparrow$ & $\mathrm{O}$ & $\uparrow$ & $\uparrow$ & $\uparrow$ \\
\hline 5years 5months & $\uparrow$ & $\uparrow$ & $\uparrow$ & $\uparrow$ & $\uparrow$ & $\uparrow$ & $\uparrow$ & $\uparrow$ & $\uparrow$ & $\uparrow$ & $\uparrow$ & $\uparrow$ & $\uparrow$ & $\uparrow$ & $\uparrow$ & $\uparrow$ & $\uparrow$ \\
\hline 5 years 6 months & 1,5 & $\uparrow$ & $\uparrow$ & $\uparrow$ & $\uparrow$ & $\uparrow$ & $\uparrow$ & $\uparrow$ & $\uparrow$ & $\uparrow$ & $\uparrow$ & $\uparrow$ & $\uparrow$ & $\times$ & $\uparrow$ & $\uparrow$ & $\uparrow$ \\
\hline 5years 7months & $\uparrow$ & $\uparrow$ & $\uparrow$ & $\uparrow$ & $\uparrow$ & $\uparrow$ & $\uparrow$ & $\uparrow$ & $\uparrow$ & $\uparrow$ & $\uparrow$ & $\uparrow$ & $\uparrow$ & $\mathrm{O}$ & $\uparrow$ & $\uparrow$ & $\uparrow$ \\
\hline 5years 8months & 1 & $\uparrow$ & $\uparrow$ & $\uparrow$ & $\uparrow$ & $\uparrow$ & $\uparrow$ & $\uparrow$ & $\uparrow$ & $\uparrow$ & $\uparrow$ & $\uparrow$ & $\uparrow$ & $\uparrow$ & $\uparrow$ & $\uparrow$ & $\uparrow$ \\
\hline 5years 9months & $\uparrow$ & $\uparrow$ & $\uparrow$ & $\uparrow$ & $\uparrow$ & $\uparrow$ & $\uparrow$ & $\uparrow$ & $\uparrow$ & $\uparrow$ & $\uparrow$ & $\uparrow$ & $\uparrow$ & $\uparrow$ & $\uparrow$ & $\uparrow$ & $\uparrow$ \\
\hline 5years 10months & $\uparrow$ & $\uparrow$ & $\uparrow$ & $\uparrow$ & $\uparrow$ & $\uparrow$ & $\uparrow$ & $\uparrow$ & $\uparrow$ & $\uparrow$ & $\uparrow$ & $\uparrow$ & $\uparrow$ & $\uparrow$ & $\uparrow$ & $\uparrow$ & $\uparrow$ \\
\hline 5years 11 months & $\uparrow$ & $\uparrow$ & $\uparrow$ & $\uparrow$ & $\uparrow$ & $\uparrow$ & $\uparrow$ & $\uparrow$ & $\uparrow$ & $\uparrow$ & $\uparrow$ & $\uparrow$ & $\uparrow$ & $\uparrow$ & $\uparrow$ & $\uparrow$ & $\uparrow$ \\
\hline 6years 0month & $\uparrow$ & $\uparrow$ & $\uparrow$ & $\uparrow$ & $\uparrow$ & $\uparrow$ & $\uparrow$ & $\uparrow$ & $\uparrow$ & $\uparrow$ & $\uparrow$ & $\uparrow$ & $\uparrow$ & $\uparrow$ & $\uparrow$ & $\uparrow$ & $\uparrow$ \\
\hline 6years 1month & 1,5 & $\uparrow$ & $\uparrow$ & $\uparrow$ & $\uparrow$ & $\uparrow$ & $\uparrow$ & $\uparrow$ & $\uparrow$ & $\uparrow$ & $\uparrow$ & $\uparrow$ & $\uparrow$ & $\uparrow$ & $\uparrow$ & $\uparrow$ & $\uparrow$ \\
\hline 6years 2months & $\uparrow$ & $\uparrow$ & $\uparrow$ & $\uparrow$ & $\uparrow$ & $\uparrow$ & $\uparrow$ & $\uparrow$ & $\uparrow$ & $\uparrow$ & $\uparrow$ & $\uparrow$ & $\uparrow$ & $\uparrow$ & $\uparrow$ & $\uparrow$ & $\uparrow$ \\
\hline 6years 3months & $\uparrow$ & $\uparrow$ & $\uparrow$ & $\uparrow$ & $\uparrow$ & $\uparrow$ & $\uparrow$ & $\uparrow$ & $\uparrow$ & $\uparrow$ & $\uparrow$ & $\uparrow$ & $\uparrow$ & $\uparrow$ & $\uparrow$ & $\uparrow$ & $\uparrow$ \\
\hline 6years 4 months & $\uparrow$ & $\uparrow$ & $\uparrow$ & $\uparrow$ & $\uparrow$ & $\uparrow$ & $\uparrow$ & $\uparrow$ & $\uparrow$ & $\uparrow$ & $\uparrow$ & $\uparrow$ & $\uparrow$ & $\uparrow$ & $\uparrow$ & $\uparrow$ & $\uparrow$ \\
\hline 6years 5months & $\uparrow$ & $\uparrow$ & $\uparrow$ & $\uparrow$ & $\uparrow$ & $\uparrow$ & $\uparrow$ & $\uparrow$ & $\uparrow$ & $\uparrow$ & $\uparrow$ & $\uparrow$ & $\uparrow$ & $\uparrow$ & $\uparrow$ & $\uparrow$ & $\uparrow$ \\
\hline
\end{tabular}


Table 3 (continued). Results of the mathematics quiz G-1

\begin{tabular}{|c|c|c|c|c|c|c|c|c|c|c|c|}
\hline & \multicolumn{11}{|c|}{ G-1 } \\
\hline & triangle & oval & trapezoid & parallelogram & rhombus & square & rectangle & pentagon & hexagon & octagon & circle \\
\hline 3years 0month & 0 & 0 & 0 & 0 & $\triangle$ & 0 & $\triangle$ & $\triangle$ & $\triangle$ & $\triangle$ & \\
\hline 3years 1month & $\uparrow$ & $\uparrow$ & $\uparrow$ & $\uparrow$ & $\uparrow$ & $\triangle$ & $\bar{\uparrow}$ & 0 & 0 & 0 & \\
\hline 3years 2months & $\uparrow$ & $\uparrow$ & $\uparrow$ & $\uparrow$ & 0 & $\mathrm{O}$ & $\uparrow$ & $\uparrow$ & $\uparrow$ & $\uparrow$ & \\
\hline 3years 3months & $\uparrow$ & $\uparrow$ & $\uparrow$ & $\uparrow$ & $\uparrow$ & $\uparrow$ & 0 & $\uparrow$ & $\uparrow$ & $\uparrow$ & \\
\hline 3years 4months & $\uparrow$ & $\uparrow$ & $\uparrow$ & $\uparrow$ & $\uparrow$ & $\uparrow$ & $\triangle$ & $\uparrow$ & $\uparrow$ & $\uparrow$ & 4 \\
\hline 3years 5months & $\uparrow$ & $\uparrow$ & $\uparrow$ & $\uparrow$ & $\uparrow$ & $\uparrow$ & $\bar{\uparrow}$ & $\uparrow$ & $\uparrow$ & $\uparrow$ & 3 \\
\hline 3years 6months & $\uparrow$ & $\uparrow$ & $\uparrow$ & $\uparrow$ & $\uparrow$ & $\uparrow$ & $\uparrow$ & $\uparrow$ & $\uparrow$ & $\uparrow$ & 0 \\
\hline 3years 7months & $\uparrow$ & $\uparrow$ & $\uparrow$ & $\uparrow$ & $\uparrow$ & $\uparrow$ & $\uparrow$ & $\uparrow$ & $\uparrow$ & $\uparrow$ & $\uparrow$ \\
\hline 3years 8months & $\uparrow$ & $\uparrow$ & $\uparrow$ & $\triangle$ & $\uparrow$ & $\uparrow$ & 0 & $\uparrow$ & $\uparrow$ & $\uparrow$ & $\uparrow$ \\
\hline 3years 9months & $\uparrow$ & $\uparrow$ & $\uparrow$ & 0 & $\uparrow$ & $\uparrow$ & $\triangle$ & $\uparrow$ & $\uparrow$ & $\uparrow$ & $\uparrow$ \\
\hline 3years 10months & $\uparrow$ & $\uparrow$ & $\uparrow$ & $\uparrow$ & $\uparrow$ & $\uparrow$ & $\mathrm{O}$ & $\uparrow$ & $\uparrow$ & $\uparrow$ & $\uparrow$ \\
\hline 3years 11months & $\uparrow$ & $\uparrow$ & $\uparrow$ & $\uparrow$ & $\uparrow$ & $\uparrow$ & $\uparrow$ & $\uparrow$ & $\uparrow$ & $\uparrow$ & $\uparrow$ \\
\hline 4years 0month & $\uparrow$ & $\uparrow$ & $\uparrow$ & $\uparrow$ & $\uparrow$ & $\uparrow$ & $\uparrow$ & $\uparrow$ & $\uparrow$ & $\uparrow$ & $\uparrow$ \\
\hline 4years 1month & $\uparrow$ & $\uparrow$ & $\uparrow$ & $\uparrow$ & $\uparrow$ & $\uparrow$ & $\uparrow$ & $\uparrow$ & $\uparrow$ & $\uparrow$ & $\uparrow$ \\
\hline 4years 2months & $\uparrow$ & $\uparrow$ & $\uparrow$ & $\uparrow$ & $\uparrow$ & $\uparrow$ & $\uparrow$ & $\uparrow$ & $\uparrow$ & $\uparrow$ & $\uparrow$ \\
\hline 4years 3months & $\uparrow$ & $\uparrow$ & $\uparrow$ & $\uparrow$ & $\uparrow$ & $\uparrow$ & $\uparrow$ & $\uparrow$ & $\uparrow$ & $\uparrow$ & $\uparrow$ \\
\hline 4years 4months & $\uparrow$ & $\uparrow$ & $\uparrow$ & $\uparrow$ & $\uparrow$ & $\uparrow$ & $\uparrow$ & $\uparrow$ & $\uparrow$ & $\uparrow$ & $\uparrow$ \\
\hline 4years 5months & $\uparrow$ & $\uparrow$ & $\uparrow$ & $\uparrow$ & $\uparrow$ & $\uparrow$ & $\uparrow$ & $\uparrow$ & $\uparrow$ & $\uparrow$ & $\uparrow$ \\
\hline 4years 6months & $\uparrow$ & $\uparrow$ & $\uparrow$ & $\uparrow$ & $\uparrow$ & $\uparrow$ & $\uparrow$ & $\uparrow$ & $\uparrow$ & $\uparrow$ & $\uparrow$ \\
\hline 4years 7months & $\uparrow$ & $\uparrow$ & $\uparrow$ & $\uparrow$ & $\uparrow$ & $\uparrow$ & $\uparrow$ & $\uparrow$ & $\uparrow$ & $\uparrow$ & $\uparrow$ \\
\hline 4years 8months & $\uparrow$ & $\uparrow$ & $\uparrow$ & $\uparrow$ & $\uparrow$ & $\uparrow$ & $\uparrow$ & $\uparrow$ & $\uparrow$ & $\uparrow$ & $\uparrow$ \\
\hline 4years 9months & $\uparrow$ & $\uparrow$ & $\uparrow$ & $\triangle$ & $\uparrow$ & $\uparrow$ & $\uparrow$ & $\uparrow$ & $\uparrow$ & $\uparrow$ & $\uparrow$ \\
\hline 4years 10months & $\uparrow$ & $\uparrow$ & $\uparrow$ & $\uparrow$ & $\uparrow$ & $\uparrow$ & $\uparrow$ & $\uparrow$ & $\uparrow$ & $\uparrow$ & $\uparrow$ \\
\hline 4years 11months & $\uparrow$ & $\uparrow$ & $\uparrow$ & $\uparrow$ & $\uparrow$ & $\uparrow$ & $\uparrow$ & $\uparrow$ & $\uparrow$ & $\uparrow$ & $\uparrow$ \\
\hline 5years 0month & $\uparrow$ & $\uparrow$ & $\Delta$ & O & $\uparrow$ & $\uparrow$ & $\triangle$ & $\uparrow$ & $\uparrow$ & $\uparrow$ & $\uparrow$ \\
\hline 5years 1month & $\uparrow$ & $\uparrow \uparrow$ & $\uparrow$ & $\uparrow$ & $\uparrow$ & $\uparrow$ & $\mathrm{O}$ & $\uparrow$ & $\uparrow$ & $\uparrow$ & $\uparrow$ \\
\hline 5years 2months & $\uparrow$ & $\uparrow$ & $\mathrm{O}$ & $\uparrow$ & $\uparrow$ & $\uparrow$ & $\uparrow$ & $\uparrow$ & $\uparrow$ & $\uparrow$ & $\uparrow$ \\
\hline 5years 3months & $\uparrow$ & $\uparrow$ & $\uparrow$ & $\uparrow$ & $\uparrow$ & $\uparrow$ & $\uparrow$ & $\uparrow$ & $\uparrow$ & $\uparrow$ & $\uparrow$ \\
\hline 5years 4months & $\uparrow$ & $\uparrow$ & $\uparrow$ & $\uparrow$ & $\uparrow$ & $\uparrow$ & $\uparrow$ & $\uparrow$ & $\uparrow$ & $\uparrow$ & $\uparrow$ \\
\hline 5years 5months & $\uparrow$ & $\uparrow$ & $\uparrow$ & $\uparrow$ & $\uparrow$ & $\uparrow$ & $\uparrow$ & $\uparrow$ & $\uparrow$ & $\uparrow$ & $\uparrow$ \\
\hline 5years 6months & $\uparrow$ & $\uparrow$ & $\uparrow$ & $\uparrow$ & $\uparrow$ & $\uparrow$ & $\uparrow$ & $\uparrow$ & $\uparrow$ & $\uparrow$ & $\uparrow$ \\
\hline 5years 7months & $\uparrow$ & $\uparrow$ & $\uparrow$ & $\uparrow$ & $\uparrow$ & $\uparrow$ & $\uparrow$ & $\uparrow$ & $\uparrow$ & $\uparrow$ & $\uparrow$ \\
\hline 5years 8months & $\uparrow$ & $\uparrow$ & $\uparrow$ & $\uparrow$ & $\uparrow$ & $\uparrow$ & $\uparrow$ & $\uparrow$ & $\uparrow$ & $\uparrow$ & $\uparrow$ \\
\hline 5years 9months & $\uparrow$ & $\uparrow$ & $\uparrow$ & $\uparrow$ & $\uparrow$ & $\uparrow$ & $\uparrow$ & $\uparrow$ & $\uparrow$ & $\uparrow$ & $\uparrow$ \\
\hline 5years 10months & $\uparrow$ & $\uparrow$ & $\uparrow$ & $\uparrow$ & $\uparrow$ & $\uparrow$ & $\uparrow$ & $\uparrow$ & $\uparrow$ & $\uparrow$ & $\uparrow$ \\
\hline 5years 11 months & $\uparrow$ & $\uparrow$ & $\uparrow$ & $\uparrow$ & $\uparrow$ & $\uparrow$ & $\uparrow$ & $\uparrow$ & $\uparrow$ & $\uparrow$ & $\uparrow$ \\
\hline 6years 0month & $\uparrow$ & $\uparrow$ & $\uparrow$ & $\uparrow$ & $\uparrow$ & $\uparrow$ & $\uparrow$ & $\uparrow$ & $\uparrow$ & $\uparrow$ & $\uparrow$ \\
\hline 6years 1month & $\uparrow$ & $\uparrow$ & $\uparrow$ & $\uparrow$ & $\uparrow$ & $\uparrow$ & $\uparrow$ & $\uparrow$ & $\uparrow$ & $\uparrow$ & $\uparrow$ \\
\hline 6years 2months & $\uparrow$ & $\uparrow$ & $\uparrow$ & $\uparrow$ & $\uparrow$ & $\uparrow$ & $\uparrow$ & $\uparrow$ & $\uparrow$ & $\uparrow$ & $\uparrow$ \\
\hline 6years 3months & $\uparrow$ & $\uparrow$ & $\uparrow$ & $\uparrow$ & $\uparrow$ & $\uparrow$ & $\uparrow$ & $\uparrow$ & $\uparrow$ & $\uparrow$ & $\uparrow$ \\
\hline 6years 4months & $\uparrow$ & $\uparrow$ & $\uparrow$ & $\uparrow$ & $\uparrow$ & $\uparrow$ & $\uparrow$ & $\uparrow$ & $\uparrow$ & $\uparrow$ & $\uparrow$ \\
\hline 6years 5months & $\uparrow$ & $\uparrow$ & $\uparrow$ & $\uparrow$ & $\uparrow$ & $\uparrow$ & $\uparrow$ & $\uparrow$ & $\uparrow$ & $\uparrow$ & $\uparrow$ \\
\hline
\end{tabular}


Table 3 (continued). Results of the mathematics quiz

\begin{tabular}{|c|c|c|c|c|c|c|c|c|c|c|c|c|c|}
\hline & \multicolumn{9}{|c|}{ G-2 } & \multirow[t]{2}{*}{ G-3 } & \multirow[t]{2}{*}{ G-4 } & \multirow[t]{2}{*}{ G-5 } & \multirow[t]{2}{*}{ G-6 } \\
\hline & $\frac{8}{3}$ & 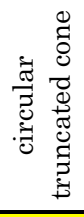 & 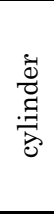 & : & 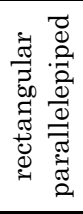 & 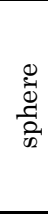 & 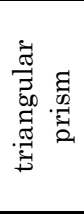 & 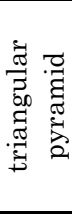 & 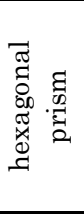 & & & & \\
\hline 3years 0month & 0 & 0 & $x$ & $x$ & $x$ & 0 & 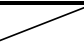 & & & & & $x$ & \\
\hline 3years 1month & $\uparrow$ & $\uparrow$ & $\uparrow$ & $\uparrow$ & $\uparrow$ & $\uparrow \uparrow$ & $x$ & & & & 7 & $\uparrow$ & \\
\hline 3years 2months & 4 & 7 & 7 & 7 & 7 & 7 & 7 & & & & & $\uparrow$ & \\
\hline 3years 3months & 0 & $\mathrm{O}$ & $x$ & $x$ & $x$ & $x$ & $x$ & & & & 4 & $\bar{\uparrow}$ & \\
\hline 3years 4months & $\uparrow$ & $\uparrow$ & $\mathrm{O}$ & $\uparrow$ & $\uparrow$ & $\mathrm{O}$ & $\uparrow$ & 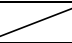 & & $\mathrm{O}$ & $\mathrm{O}$ & $\uparrow$ & \\
\hline 3years 5months & $\uparrow$ & $\uparrow$ & $\uparrow$ & $\uparrow$ & $\uparrow$ & $\uparrow$ & $\uparrow$ & $\mathrm{O}$ & & $\uparrow$ & $\uparrow$ & $\uparrow$ & \\
\hline 3years 6months & $\uparrow$ & $\uparrow$ & $\uparrow$ & $\uparrow$ & $\triangle$ & $\uparrow$ & $\uparrow$ & $\uparrow$ & & $\uparrow$ & $\uparrow$ & $\uparrow$ & 0 \\
\hline 3years 7 months & $\uparrow$ & $\uparrow$ & $\uparrow$ & $\uparrow$ & $x$ & $\uparrow$ & $\uparrow$ & $\uparrow$ & & $\uparrow$ & $\uparrow$ & $\uparrow$ & $\uparrow$ \\
\hline 3years 8months & $\uparrow$ & $\uparrow$ & $\uparrow$ & 0 & $\uparrow$ & $\uparrow$ & $\uparrow$ & $\uparrow$ & 7 & $\uparrow$ & $\uparrow$ & $\uparrow$ & $\uparrow$ \\
\hline 3years 9months & $\uparrow$ & $\uparrow$ & $\uparrow$ & $\uparrow$ & $\uparrow$ & $\uparrow$ & $\uparrow$ & $\uparrow$ & 7 & $\uparrow$ & $\uparrow$ & 0 & $\uparrow$ \\
\hline 3years 10months & $\uparrow$ & $\uparrow$ & $\uparrow$ & $x$ & O & $\uparrow$ & $\uparrow$ & $\uparrow$ & 0 & $\uparrow$ & $\uparrow$ & $\uparrow$ & $\uparrow$ \\
\hline 3years 11months & $\uparrow$ & $\uparrow$ & $\uparrow$ & $\mathrm{O}$ & $\Delta$ & $\uparrow$ & $\Delta$ & $\uparrow$ & $\Delta$ & $\uparrow$ & $\uparrow$ & $x$ & $\uparrow$ \\
\hline 4years 0month & $\uparrow$ & $\uparrow$ & $\uparrow$ & $\triangle$ & $\mathrm{O}$ & $\triangle$ & $\uparrow$ & $\Delta$ & $\mathrm{O}$ & $\uparrow$ & $\uparrow$ & $\uparrow$ & $\uparrow$ \\
\hline 4years 1month & $\uparrow$ & $\uparrow$ & $\uparrow$ & $\mathrm{O}$ & $\uparrow$ & $\mathrm{O}$ & $x$ & $\mathrm{O}$ & $x$ & $\uparrow$ & $\uparrow$ & $\uparrow$ & $\uparrow$ \\
\hline 4years 2months & $\uparrow$ & $\uparrow$ & $\uparrow$ & $x$ & $\uparrow$ & $\uparrow$ & $\uparrow$ & $\uparrow$ & O & $\uparrow$ & $\uparrow$ & $\mathrm{O}$ & $\uparrow$ \\
\hline 4years 3months & $\uparrow$ & $\uparrow$ & $\uparrow$ & $\mathrm{O}$ & $\uparrow$ & $\uparrow$ & $\uparrow$ & $\Delta$ & $\uparrow$ & $\uparrow$ & $\uparrow$ & $x$ & $\uparrow$ \\
\hline 4years 4months & $\uparrow$ & $\uparrow$ & $\uparrow$ & $\uparrow$ & $\uparrow$ & $\uparrow$ & $\uparrow$ & $\mathrm{O}$ & $\uparrow$ & $\uparrow$ & $\uparrow$ & $\uparrow$ & $\uparrow$ \\
\hline 4years 5months & $\uparrow$ & $\uparrow$ & $\uparrow$ & $\uparrow$ & $\uparrow$ & $\uparrow$ & 0 & $\uparrow$ & $\uparrow$ & $\uparrow$ & $\uparrow$ & $\uparrow$ & $\uparrow$ \\
\hline 4years 6months & $\uparrow$ & $\uparrow$ & $\Delta$ & $\uparrow$ & $\uparrow$ & $\uparrow$ & $\triangle$ & $\uparrow$ & $\Delta$ & $\uparrow$ & $\uparrow$ & $\uparrow$ & $\uparrow$ \\
\hline 4years 7months & $\uparrow$ & $\uparrow$ & $\mathrm{O}$ & $\uparrow$ & $\Delta$ & $\uparrow$ & $\uparrow$ & $\triangle$ & O & $\uparrow$ & $\uparrow$ & $\uparrow$ & $\uparrow$ \\
\hline 4years 8months & $\uparrow$ & $\uparrow$ & $\uparrow$ & $\triangle$ & O & $\uparrow$ & $x$ & $x$ & $\Delta$ & $\uparrow$ & $\uparrow$ & $\uparrow$ & $\uparrow$ \\
\hline 4years 9months & $\triangle$ & $\uparrow$ & $\uparrow$ & $x$ & $\triangle$ & $\uparrow$ & $\triangle$ & $\Delta$ & $\triangle$ & $\uparrow$ & $\uparrow$ & $\uparrow$ & $\uparrow$ \\
\hline 4years 10months & $\mathrm{O}$ & $\triangle$ & $\uparrow$ & $\uparrow$ & $\uparrow$ & $\triangle$ & $\uparrow$ & $\uparrow$ & $\uparrow$ & $\uparrow$ & $\uparrow$ & 0 & $\uparrow$ \\
\hline 4years 11months & $\uparrow$ & $\mathrm{O}$ & $\Delta$ & $\uparrow$ & $\uparrow$ & $\mathrm{O}$ & 0 & 0 & $\uparrow$ & $\uparrow$ & $\uparrow$ & $\uparrow$ & $\uparrow$ \\
\hline 5years 0month & $\uparrow$ & $\uparrow$ & $\mathrm{O}$ & $\triangle$ & $\triangle$ & $\uparrow$ & $\Delta$ & $\triangle$ & $\uparrow$ & $\uparrow$ & $\uparrow$ & $\uparrow$ & $\uparrow$ \\
\hline 5years 1month & $\uparrow$ & $\uparrow$ & $\uparrow$ & $\mathrm{O}$ & $\uparrow$ & $\uparrow$ & $\mathrm{O}$ & $\mathrm{O}$ & $\mathrm{O}$ & $\uparrow$ & $\uparrow$ & $\bar{\uparrow}$ & $\uparrow$ \\
\hline 5years 2months & $\uparrow$ & $\uparrow$ & $\uparrow$ & $\uparrow$ & $\uparrow$ & $\Delta$ & $\uparrow$ & $\triangle$ & $\triangle$ & $\uparrow$ & $\uparrow$ & $\uparrow$ & $\uparrow$ \\
\hline 5years 3months & $\uparrow$ & $\uparrow$ & $\uparrow$ & $\uparrow$ & $\uparrow$ & $\mathrm{O}$ & $\uparrow$ & $\mathrm{O}$ & $\uparrow$ & $\uparrow$ & $\uparrow$ & $\uparrow$ & $\uparrow$ \\
\hline 5years 4months & $\uparrow$ & $\uparrow$ & $\uparrow$ & $\uparrow$ & 0 & $\Delta$ & $\Delta$ & $\uparrow$ & $\uparrow$ & $\uparrow$ & $\uparrow$ & $\uparrow$ & $\uparrow$ \\
\hline 5years 5months & $\uparrow$ & $\uparrow$ & $\uparrow$ & $x$ & $\Delta$ & $\mathrm{O}$ & $\uparrow$ & $x$ & $\mathrm{O}$ & $\uparrow$ & $\uparrow$ & $\uparrow$ & $\uparrow$ \\
\hline 5years 6months & $\uparrow$ & $\uparrow$ & $\uparrow$ & $\mathrm{O}$ & $\uparrow$ & $\uparrow$ & $\uparrow$ & $\Delta$ & $\uparrow$ & $\uparrow$ & $\uparrow$ & $\uparrow$ & $\uparrow$ \\
\hline 5years 7months & $\uparrow$ & $\uparrow$ & $\triangle$ & $\uparrow$ & 0 & $\uparrow$ & $\uparrow$ & $x$ & $\triangle$ & $\uparrow$ & $\uparrow$ & $\uparrow$ & $\uparrow$ \\
\hline 5years 8months & $\uparrow$ & $\triangle$ & $\mathrm{O}$ & $\uparrow$ & $\uparrow$ & $\uparrow$ & $\mathrm{O}$ & $\mathrm{O}$ & $\mathrm{O}$ & $\uparrow$ & $\uparrow$ & $\uparrow$ & $\uparrow$ \\
\hline 5years 9months & $\uparrow$ & $\mathrm{O}$ & $\uparrow$ & $\triangle$ & $\uparrow$ & $\uparrow$ & $\uparrow$ & $\Delta$ & $\uparrow$ & $\uparrow$ & $\uparrow$ & $\uparrow$ & $\uparrow$ \\
\hline 5years 10 months & $\uparrow$ & $\uparrow$ & $\uparrow$ & $\uparrow$ & $\uparrow$ & $\uparrow$ & $\uparrow$ & $\mathrm{O}$ & $\uparrow$ & $\uparrow$ & $\uparrow$ & $\uparrow$ & $\uparrow$ \\
\hline 5years 11months & $\uparrow$ & $\Delta$ & $\uparrow$ & $\mathrm{O}$ & $\uparrow$ & $\uparrow$ & $\uparrow$ & $\uparrow$ & $\uparrow$ & $\uparrow$ & $\uparrow$ & $\uparrow$ & $\uparrow$ \\
\hline 6years 0month & $\uparrow$ & $\mathrm{O}$ & $\triangle$ & $\triangle$ & $\uparrow$ & $\uparrow$ & $\uparrow$ & $\uparrow$ & $\uparrow$ & $\uparrow$ & $\uparrow$ & $\uparrow$ & $\uparrow$ \\
\hline 6years 1month & $\uparrow$ & $\uparrow$ & $\uparrow$ & $\mathrm{O}$ & $\uparrow$ & $\uparrow$ & $\uparrow$ & $\uparrow$ & $\uparrow$ & $\uparrow$ & $\uparrow$ & $\uparrow$ & $\uparrow$ \\
\hline 6years 2months & $\uparrow$ & $\Delta$ & $\uparrow$ & $\triangle$ & $\triangle$ & $\uparrow$ & $\uparrow$ & $\uparrow$ & $\uparrow$ & $\uparrow$ & $\uparrow$ & $\uparrow$ & $\uparrow$ \\
\hline 6years 3months & $\uparrow$ & O & $x$ & $x$ & $\mathrm{O}$ & $\uparrow$ & $\uparrow$ & $\uparrow$ & $\uparrow$ & $\uparrow$ & $\uparrow$ & $\uparrow$ & $\uparrow$ \\
\hline 6years 4months & $\uparrow$ & $\bar{\uparrow}$ & $\mathrm{O}$ & $\triangle$ & $\uparrow$ & $\uparrow$ & $\uparrow$ & $\uparrow$ & $\uparrow$ & $\uparrow$ & $\uparrow$ & $\bar{\uparrow}$ & $\uparrow$ \\
\hline 6years 5months & $\uparrow$ & $\bar{\uparrow}$ & $\uparrow$ & $\mathrm{O}$ & $\uparrow$ & $\bar{\uparrow}$ & $\uparrow$ & $\uparrow$ & $\uparrow$ & $\uparrow$ & $\uparrow$ & $\uparrow$ & $\uparrow$ \\
\hline
\end{tabular}




\section{Watanabe}

Table 3 (continued). Results of the mathematics quiz

\begin{tabular}{|c|c|c|c|c|c|c|c|c|c|c|c|c|c|}
\hline & \multicolumn{8}{|c|}{ G-7 } & \multicolumn{4}{|c|}{ G-8 } & \multirow[t]{2}{*}{ G-9 } \\
\hline & circle & oval & triangle & rectangle & squere & trapezoid & rhombus & $\begin{array}{c}\text { parallelog } \\
\text { ram }\end{array}$ & ruler & $\begin{array}{r}\text { triangul } \\
\text { scale }\end{array}$ & $\begin{array}{l}\text { triangular } \\
\text { ruler }\end{array}$ & compass & \\
\hline 3years 0month & $\mathrm{O}$ & 0 & $\bar{\triangle}$ & & & & & & 0 & & & & \\
\hline 3years 1month & $\uparrow$ & $\uparrow$ & 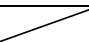 & $\gamma$ & & & & & 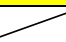 & 7 & 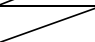 & & \\
\hline 3years 2months & $\uparrow$ & $\uparrow$ & & & & & & & $\mathrm{O}$ & 0 & & - & \\
\hline 3years 3months & $\uparrow$ & $\uparrow$ & $\Delta$ & $\triangle$ & & & & & 7 & $\uparrow$ & 0 & 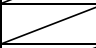 & \\
\hline 3years 4months & $\uparrow$ & $\uparrow$ & $\uparrow$ & $x$ & & & & & 7 & $\uparrow$ & $\uparrow$ & 7 & \\
\hline 3years 5months & $\uparrow$ & $\uparrow$ & O & $\triangle$ & & & & & O & $\uparrow$ & $\uparrow$ & $\triangle$ & \\
\hline 3years 6months & $\uparrow$ & $\uparrow$ & $\triangle$ & $\uparrow$ & & & & & $\uparrow$ & $\uparrow$ & $\uparrow$ & $\uparrow$ & \\
\hline 3years 7months & $\uparrow$ & $\uparrow$ & & & & & & & $\uparrow$ & $\uparrow$ & $\uparrow$ & $\uparrow$ & \\
\hline 3years 8months & $\uparrow$ & $\uparrow$ & 7 & $\gamma$ & & & & & $\uparrow$ & $\uparrow$ & 1 & $\uparrow$ & \\
\hline 3years 9months & $\uparrow$ & $\uparrow$ & $\mathrm{O}$ & 0 & & & & & $\uparrow$ & $\uparrow$ & 7 & $\uparrow$ & \\
\hline 3years 10months & $\uparrow$ & $\uparrow$ & $\uparrow$ & $\uparrow$ & 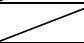 & & & & $\uparrow$ & 7 & $\mathrm{O}$ & $\uparrow$ & \\
\hline 3years 11months & $\uparrow$ & $\uparrow$ & $\uparrow$ & $\uparrow$ & 4 & 4 & & & $\uparrow$ & $\mathrm{O}$ & $\uparrow$ & $\uparrow$ & \\
\hline 4years 0month & $\uparrow$ & $\uparrow$ & $\uparrow$ & $\bar{\uparrow}$ & $\mathrm{O}$ & & & & $\bar{\uparrow}$ & $\bar{\uparrow}$ & $\uparrow$ & $\bar{\uparrow}$ & \\
\hline 4years 1month & $\uparrow$ & $\uparrow$ & $\uparrow$ & $\uparrow$ & 7 & & & & $\uparrow$ & $\uparrow$ & 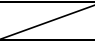 & $\uparrow$ & \\
\hline 4years 2months & $\uparrow$ & $\uparrow$ & $\uparrow$ & $\uparrow$ & 0 & & & & $\uparrow$ & $\uparrow$ & 0 & $\uparrow$ & \\
\hline 4years 3months & $\uparrow$ & $\uparrow$ & $\uparrow$ & $\uparrow$ & $\uparrow$ & & & & $\uparrow$ & $\uparrow$ & $\uparrow$ & $\uparrow$ & \\
\hline 4years 4months & $\uparrow$ & $\uparrow$ & $\uparrow$ & $\uparrow$ & $\uparrow$ & & & & $\uparrow$ & $\uparrow$ & 1 & $\uparrow$ & \\
\hline 4 years 5months & $\uparrow$ & $\uparrow$ & $\uparrow$ & $\uparrow$ & $\uparrow$ & & & & $\bar{\uparrow}$ & $\uparrow$ & O & $\uparrow$ & cube \\
\hline 4years 6months & $\uparrow$ & $\uparrow$ & $\uparrow$ & $\uparrow$ & $\uparrow$ & & & & $\uparrow$ & $\uparrow$ & $\uparrow$ & $\uparrow$ & $\uparrow$ \\
\hline 4years 7months & $\uparrow$ & $\uparrow$ & $\uparrow$ & $\uparrow$ & $\uparrow$ & & & & $\uparrow$ & $\uparrow$ & $\uparrow$ & $\uparrow$ & $\uparrow$ \\
\hline 4 years 8months & $\uparrow$ & $\uparrow$ & $\uparrow$ & $\uparrow$ & $\uparrow$ & & & & $\uparrow$ & $\uparrow$ & $\uparrow$ & $\uparrow$ & $\uparrow$ \\
\hline 4years 9months & $\uparrow$ & $\uparrow$ & $\uparrow$ & $\uparrow$ & $\uparrow$ & 4 & & & $\uparrow$ & $\uparrow$ & $\uparrow$ & $\uparrow$ & $\uparrow$ \\
\hline 4years 10months & $\uparrow$ & $\uparrow$ & $\uparrow$ & $\uparrow$ & $\uparrow$ & 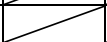 & & 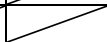 & $\uparrow$ & $\uparrow$ & $\uparrow$ & $\uparrow$ & $\uparrow$ \\
\hline 4 years 11months & $\uparrow$ & $\uparrow$ & $\uparrow$ & $\uparrow$ & $\uparrow$ & 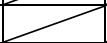 & & 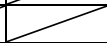 & $\uparrow$ & $\uparrow$ & $\uparrow$ & $\uparrow$ & $\uparrow$ \\
\hline 5years 0month & $\uparrow$ & $\uparrow$ & $\uparrow$ & $\uparrow$ & $\uparrow$ & 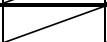 & & & $\bar{\uparrow}$ & $\uparrow$ & $\uparrow$ & $\bar{\uparrow}$ & $\uparrow$ \\
\hline 5years 1month & $\uparrow$ & $\uparrow$ & $\uparrow$ & $\uparrow$ & $\uparrow$ & 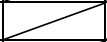 & & & $\uparrow$ & $\uparrow$ & $\uparrow$ & $\uparrow$ & $\uparrow$ \\
\hline 5years 2months & $\uparrow$ & $\uparrow$ & $\uparrow$ & $\uparrow$ & $\uparrow$ & & & & $\uparrow$ & $\uparrow$ & $\uparrow$ & $\uparrow$ & $\uparrow$ \\
\hline 5years 3months & $\uparrow$ & $\uparrow$ & $\uparrow$ & $\uparrow$ & $\uparrow$ & & & & $\uparrow$ & $\uparrow$ & $\uparrow$ & $\uparrow$ & $\uparrow$ \\
\hline 5years 4months & $\uparrow$ & $\uparrow$ & $\uparrow$ & $\uparrow$ & $\uparrow$ & $\angle$ & & 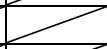 & $\uparrow$ & $\uparrow$ & $\uparrow$ & $\uparrow$ & $\uparrow$ \\
\hline 5years 5months & $\uparrow$ & $\uparrow$ & $\uparrow$ & $\uparrow$ & $\uparrow$ & 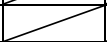 & & 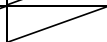 & $\uparrow$ & $\uparrow$ & $\uparrow$ & $\uparrow$ & $\uparrow$ \\
\hline 5years 6months & $\uparrow$ & $\uparrow$ & $\uparrow$ & $\uparrow$ & $\uparrow$ & 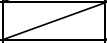 & 4 & 4 & $\uparrow$ & $\uparrow$ & $\uparrow$ & $\uparrow$ & $\uparrow$ \\
\hline 5years 7months & $\uparrow$ & $\uparrow$ & $\uparrow$ & $\uparrow$ & $\uparrow$ & $\triangle$ & $\triangle$ & $\triangle$ & $\uparrow$ & $\uparrow$ & $\uparrow$ & $\uparrow$ & $\uparrow$ \\
\hline 5years 8months & $\uparrow$ & $\uparrow$ & $\uparrow$ & $\uparrow$ & $\uparrow$ & $x$ & 0 & $x$ & $\uparrow$ & $\uparrow$ & $\uparrow$ & 0 & $\uparrow$ \\
\hline 5years 9months & $\uparrow$ & $\uparrow$ & $\uparrow$ & $\uparrow$ & $\uparrow$ & 0 & $\uparrow$ & $\uparrow$ & $\uparrow$ & $\uparrow$ & $\uparrow$ & $\uparrow$ & $\uparrow$ \\
\hline 5years 10months & $\uparrow$ & $\uparrow$ & $\uparrow$ & $\uparrow$ & $\uparrow$ & $\uparrow$ & $\uparrow$ & 0 & $\uparrow$ & $\uparrow$ & $\uparrow$ & $\uparrow$ & $\uparrow$ \\
\hline 5years 11months & $\uparrow$ & $\uparrow$ & $\uparrow$ & $\uparrow$ & $\uparrow$ & $\uparrow$ & $\uparrow$ & $\uparrow$ & $\uparrow$ & $\uparrow$ & $\uparrow$ & $\uparrow$ & $\uparrow$ \\
\hline 6years 0month & $\uparrow$ & $\uparrow$ & $\bar{\uparrow}$ & $\uparrow$ & $\bar{\uparrow}$ & $\uparrow$ & O & $\triangle$ & $\uparrow$ & $\bar{\uparrow}$ & $\uparrow$ & $\uparrow$ & $\uparrow$ \\
\hline $6 y e a r s$ 1month & $\uparrow$ & $\uparrow$ & $\uparrow$ & $\uparrow$ & $\uparrow$ & $\uparrow$ & $\uparrow$ & $x$ & $\uparrow$ & $\uparrow$ & $\uparrow$ & $\uparrow$ & \begin{tabular}{|c} 
cube \\
trigonal \\
pyramid
\end{tabular} \\
\hline 6years 2months & $\uparrow$ & $\uparrow$ & $\uparrow$ & $\uparrow$ & $\uparrow$ & $\uparrow$ & $\uparrow$ & $\uparrow$ & $\uparrow$ & $\uparrow$ & $\uparrow$ & $\uparrow$ & cube \\
\hline 6 years 3months & $\uparrow$ & $\uparrow$ & $\uparrow$ & $\uparrow$ & $\uparrow$ & $\Delta$ & $\uparrow$ & & $\uparrow$ & $\uparrow$ & $\uparrow$ & $\Delta$ & \begin{tabular}{|c|} 
cube \\
trigonal \\
pyramid \\
quadrang \\
ular \\
pyramid \\
cylinder \\
\end{tabular} \\
\hline 6years 4months & $\uparrow$ & $\uparrow$ & $\uparrow$ & $\uparrow$ & $\uparrow$ & $\mathrm{O}$ & $\uparrow$ & $\triangle$ & $\uparrow$ & $\uparrow$ & $\uparrow$ & $\uparrow$ & cube \\
\hline 6 years 5months & $\uparrow$ & $\uparrow$ & $\uparrow$ & $\uparrow$ & $\uparrow$ & $\uparrow$ & & $\uparrow$ & $\uparrow$ & $\uparrow$ & $\uparrow$ & 0 & \begin{tabular}{|c|} 
cube \\
quadrang \\
ular \\
pyramid \\
trigonal \\
pyramid \\
\end{tabular} \\
\hline
\end{tabular}


Table 3 (continued). Results of the mathematics quiz

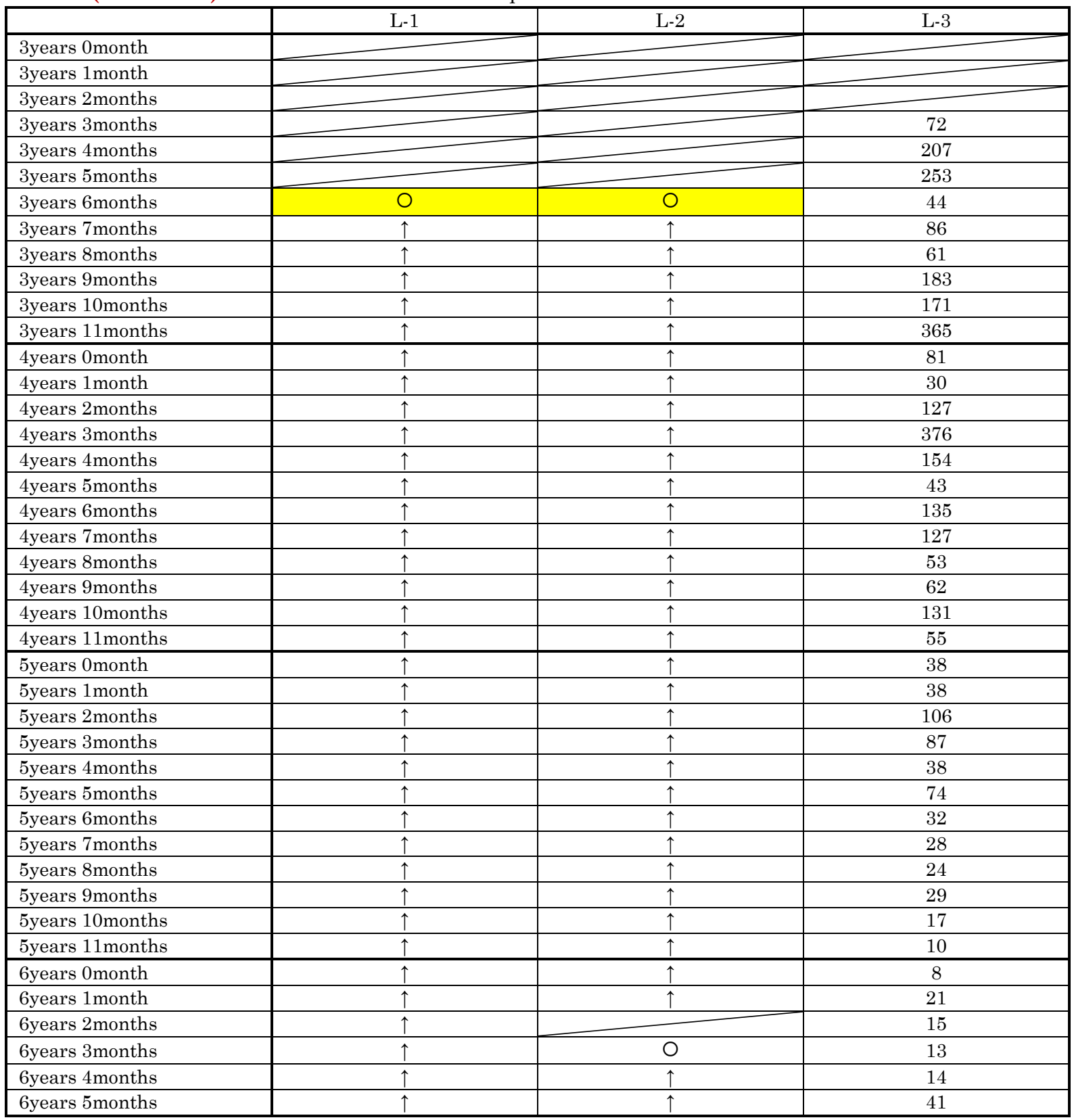

Since most of the content to be learned in primary school is content, it is reasonable to think that many contents cannot be known without experience or intentional learning. As per the obtained results, it was observed that many contents had already been understood by the participant from the age of three. It was understood that the content at the age of four and five had increased. In addition, it is suggested that there are quite a lot of items that were considered stable (continuing correct answers over three months). For the progress of the acquisition phase regarding the conservation concept, please refer to the following papers: Watanabe (2017a, 2017b).

\section{Non-cognitive Skills (Marshmallow Test and Attendance Status of Kindergarten)}

The marshmallow test was carried out in the usual manner. In both the 15-minute and 20-minute tests, at the age of five years and eight months, the participant was successful. In addition, at the age of six years and two months, the participant underwent an objective marshmallow test administered by university 
students at kindergarten. The child succeeded in 10 minutes (for reference, the success rates for this test were as follows). There are 32 subjects: four-year-olds-4/8, five-year-olds-9/17, six-year-olds-2/5 (Bamba, 2018).

The child attended the kindergarten for three years without missing a single day or being late.

\section{General Abilities (Cognitive Skills and Non-Cognitive Skills)}

This study's subject was asked by her guardian whether she would like to participate in a quiz competition for the following reason: she enjoyed attending an elementary school trial class and for two to three years had been looking forward to studying at an elementary school. She said she would definitely like to participate; thus, she took entrance examinations (she called the examinations quiz competitions.): two in October (private), two in January (national public), and one in February (private). She participated in all of these "quiz competitions" actively. Even after the quiz competitions ended, she would ask when the next one would start and whether there would be quiz competitions after entering elementary school. In other words, she looked forward to participating in them. Furthermore, when the child would see her guardian after the end of a quiz competition, she would always say that it was interesting and that she wanted to do it again. She applied to five schools and took entrance examinations at four of them (she withdrew her application from one school because of a scheduling issue). She was accepted to two of the three private schools and was not accepted to the national public school.

\section{DISCUSSION}

There are few studies on attempts to nurture both non-cognitive skills and cognitive skills simultaneously at home. There are few studies that are conscious of the systematicity and continuity of mathematical learning contents and their wide-ranging, multiple areas at home as well (Anders \& Rossbach, 2015; Carpenter, Franke, Johnson, Turrou, \& Wager, 2016; Clements \& Sarama, 2014; Tucker, 2014; Van Hoorn, Nourot, Scales, \& Alward, 2014; Verdine et al., 2014).

In many such studies, the main purpose is to train mathematical cognitive skills, while, in the case of play, systematic mathematics are often disregarded. Therefore, in this research we aimed to fuse each type of strength.

A hypothesis of this research is as follows. We can create a simple mathematics early childhood play. In addition, contents of the play can consist of wide-ranging mathematics. And young children can play at home. The play can promote non-cognitive skills and mathematical cognitive skills, because play promote noncognitive skills and contents of the play included mathematics.

As a result of the single case study, it was suggested that certain improvements in mathematical cognitive skills can be recognized and used to help acquire non-cognitive skills (self-control and GRIT) in early childhood. That is, simple mathematics play at home promotes both non-cognitive and cognitive skills in early childhood.

The above discussion suggests that the simple mathematics play at home can directly contribute to the development of non-cognitive skills (self-control and GRIT), as well as support, to an extent, the development of cognitive skills. Simple mathematics play is valuable even solely for the reason that children can engage in immersive play. In other words, the hypothesis of research is supported.

The reason for this is as follows. The child's mathematical ability (cognitive skills) can be increased by the mathematics play at home. The participant's non-cognitive skills increased due to the simple mathematics play too. She did have good non-cognitive skills and enjoyed performing many mathematics quiz questions every day. Therefore, it is possible that the quiz contributed to the development of her non-cognitive skills. Moreover, she was successful in the marshmallow test, and she attended the kindergarten for three years without missing a single day or being late. The participant was able to pass the examination. Indeed, for these quiz competitions, the child did not attend a private tutoring school, nor did she study specifically for the entrance examinations or receive related instruction. Almost all her preparation was for the mathematics quiz play, and while past entrance examination questions were incorporated into it, explanations and guidance were not provided to her. In Japan, to take an elementary school entrance examination, most people attend a private tutoring school. Passing such examinations is thought to be difficult without tutoring services. (This was also stated by the principal of one of the schools that she applied to.). In other words, being able to handle entrance examinations without undergoing early education specifically geared toward such examinations (technical guidance) indicates that she probably had already acquired cognitive skills and non-cognitive skills. (Since she did not pass all schools and was not a prodigy, this suggests that her learning had an influence.). 
For example, in the information of a cram school, the number of successful H-elementary school students is 158 (unpublished from elementary school). In the A cram school, 97 people passed the H-elementary school and 60 people passed the H-elementary school in the B cram school. In other words, 157 people went to the cram school.

Now, we discuss some awaiting solution in this study. First, it is worth noting that this was only a single case study. A single case study is the first step toward new findings, and as a disadvantage, it lacks generality. Although we can imagine easily this time, it can be inferred that this cannot be done easily in kindergartens or general households due to the large number of question items. Therefore, we adopted a single case study strategy. It seems that there was merit in finding out what kind of play is possible. Of course, lack of generality is cited as a challenge; however, now it is possible for a three year old infant to work on one year at another family, and to a certain extent (change it to once every two months etc.) it is possible we got a report.

Then, the following points must also be considered in this study. It is unlikely that simple mathematics play can heighten all the non-cognitive skills. Indeed, cognitive skills will probably not be sharpened by engaging only in simple mathematics play. It is important to see the mathematics play not only as a nuisance but as part of necessary learning. When incorporating knowledge regarding, for example, the mutual interplay of genetics and the environment, epigenetics, and probabilistic epigenesis (Johnson \& De Haan, 2015), it is important to create an environment in which children constantly come into contact with mathematics. This can be partially accomplished through the simple mathematics quiz game.

And, in Japan, there is a strong tendency toward limited cooperation for early childhood education claiming "play" and mathematics education claiming "subject education." In this research, we aimed at adopting the claims of both, and we were able to show good results. Based on this result, it is expected that this play will be easy to adopt for early childhood education. Moreover, it is likely to be useful in improving the stumbling (First-Grade Problem) in the first grade of elementary school, which is a problem in Japan.

Another benefit of this mathematics play is that the amount of time spent with the child will increase. Generally, the establishment of a trusting relationship (rapport) between the subject and researcher is a major premise of psychological tests and the like. When the person administering the questions is the child's guardian, there is no need to try to maintain rapport.

Even when the father is a questioner, the following merit must also be considered. Contact with fathers has been shown to have a positive effect on child rearing, in relationship to both cognitive and non-cognitive abilities (Hartwell-Walker, 2016; Nauert, 2015).

Furthermore, while children naturally absorb many things, only a few opportunities are present for them to produce outputs. Since it has been found that output is important (Karpicke \& Roediger, 2008), it appears that this mathematics play is ideal for this purpose.

\section{ACKNOWLEDGEMENT}

This work was supported by JSPS KAKENHI under Grant number 16K01043.

\section{Disclosure statement}

No potential conflict of interest was reported by the authors.

\section{Notes on contributors}

Nobuki Watanabe - School of Education, Kwansei Gakuin University, Japan. 


\section{REFERENCES}

Anders, Y., \& Rossbach, H. G. (2015). Preschool teachers' sensitivity to mathematics in children's play: the influence of math-related school experiences emotional attitudes, and pedagogical beliefs. Journal of Research in Childhood Education, 29(3), 305-322. https://doi.org/10.1080/02568543.2015.1040564

Asbury, K., \& Plomin, R. (2014). G is for genes: the impact of genetics on education and achievement. London: Wiley-Blackwell (pp. 144-145).

Bamba, H. (2018). Influence of birth order on self-control'. (Graduation Thesis). Kyoto University.

Barlow, D. H., Nock, M. K., \& Hersen, M. (2009). Single case experimental designs: strategies for studying behavior change. Boston: Allyn and Bacon (pp. 20-26).

Carpenter, T. P., Franke, M. L., Johnson, N. C., Turrou, A. C., \& Wager, A. A. (2016). Young children's mathematics: cognitively guided instruction in early childhood education. Portsmouth, NH: Heinemann (pp. 1-147).

Clements, D. H., \& Sarama, J. (2014). Play, mathematics, and false dichotomies. https://doi.org/10.1007/BF00230990\#page-1

Crehan, L. (2018). Clever lands. London: unbound, 1-274.

Hartwell-Walker, M. (2016). Fathers Are Not inferior parents. Psych central. Retrieved from https://psychcentral.com/lib/fathers-are-not-inferior-parents/

Heckman, J. J. (2013). Giving kids a fair chance. Cambridge, MA: MIT Press (pp. 3-41).

Hirsh-Pasek, K., \& Golinkoff, R. M. (2003). Einstein never used flashcards: how our children really learn and why they need to play more and memorize less. Emmaus, PA: Rosedale, 210-213.

Johnson, M. H., \& De Haan, M. (2015). Developmental Cognitive Neuroscience. Oxford, UK: Wiley-Blackwell, $1-42$.

Karpicke, J. D., \& Roediger, H. L. (2008). The critical importance of retrieval for learning. Science, 319(5865), 966-968. http://dx.doi.org/10.1126/science.1152408.

Klibanoff, R. S., Levine, S. C., Huttenlocher, J., Vasilyeva, M., \& Hedges, L. V. (2006). Preschool children's mathematical knowledge: the effect of teacher "math talk." developmental Psychology, 42(1), 59-69.

Marcus, G. (2004). The birth of the mind: how a tiny number of genes creates the complexities of human thought. Cambridge: Basic Books (pp. 176-177).

Ministry of Education, Culture, Sports, Science, and Technology (2017). Course of study for Kindergarten. Retrieved from http://www.mext.go.jp/component/a_menu/education/micro_detail/_icsFiles/afieldfile/ 2017/05/12/1384661_3_2.pdf

Mischel, W. (2014). The marshmallow test: mastering self-control. New York, US, 13-28.

Narita, T. (Ed.) (2010). Contents of early childhood care and education: language (second ed.), Gifu: Mirai (pp. 185-187).

Nauert, R. (2015). Active father-figure helps kids. Psych central. Retrieved from https://psychcentral.com/news/2008/02/12/active-father-figure-helps-kids/1903.html

Organization for economic co-operation and development (2015). Skills for social progress the power of social and emotional skills. Retrieved from http://www.oecd.org/education/skills-for-social-progress9789264226159-en.htm

Rutter, M. (2006). Genes and behavior: nature-nurture interplay explained. London: Blackwell Publishing (pp. 211-225).

Siraj-Blatchford, I. (2015). Effective pedagogy in the early years and the long term impact on language and math development. Paper presented at the $26^{\text {th }}$ annual meeting for. the japan society of developmental psychology, 136-137.

Spector, T. (2012). Identically different: Why you can change your genes. London: Weidenfeld \& Nicolson (pp. 289-293).

Tagami, T., \& Takaara, M. (2016). Instructional method of contents in early childhood care and education: language (new revision). Tokyo: Houbunshorin (pp. 70-74).

Tucker, K. (2014). Mathematics through play in the early years. Thousand Oaks, CA: Sage (pp. 1-144).

Van Hoorn, J., Nourot, P. M., Scales, B., \& Alward, K. R. (2014). Play at the center of the curriculum. Upper Saddle River, NJ: Pearson Higher Education. 
Verdine, B. N., Golinkoff, R. M., Hirsh-Pasek, K., Newcombe, N. S., Filipowicz, A. T., \& Chang, A. (2014). Deconstructing building blocks: preschoolers' spatial assembly performance relates to early mathematical skills. Child Development, 85(3), 1062-1076. https://doi.org/10.1111/cdev.12165

Watanabe, N. (2017a). Acquiring Piaget's conservation concept of numbers, lengths, and liquids as ordinary play. Journal of Educational and Developmental Psychology, 7(1), 210-217. https://doi.org/10.5539/jedp.v7n1p210

Watanabe, N. (2017b). Accelerated cognitive development-Piaget's conservation concept. Journal of Educational and Developmental Psychology, 7(2), 68-74. https://doi.org/10.5539/jedp.v7n2p68 